JOURNAL OF SYNCHROTRON RADIATION

ISSN 1600-5775

Received 6 March 2021

Accepted 15 July 2021

Edited by Y. Amemiya, University of Tokyo, Japan

Keywords: high energy density; X-ray free-electron lasers; warm dense matter; high-pressure science; relativistic laser-matter interaction.
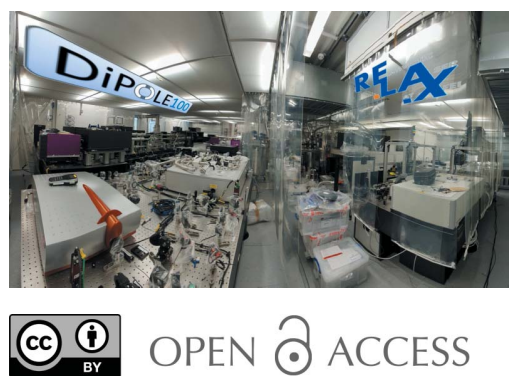

\section{The High Energy Density Scientific Instrument at the European XFEL}

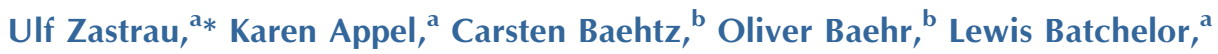
Andreas Berghäuser, ${ }^{\mathrm{b}}$ Mohammadreza Banjafar, ${ }^{\text {a,b }}$ Erik Brambrink, ${ }^{\mathrm{a}}$ Valerio Cerantola, ${ }^{a}$ Thomas E. Cowan, ${ }^{\mathrm{b}}$ Horst Damker, ${ }^{\mathrm{c}}$ Steffen Dietrich, Samuele Di Dio Cafiso, ${ }^{\mathrm{b}}$ Jörn Dreyer, ${ }^{\mathrm{b}}$ Hans-Olaf Engel, ${ }^{\mathrm{b}}$ Thomas Feldmann, ${ }^{\mathrm{a}}$ Stefan Findeisen, ${ }^{\mathrm{b}}$ Manon Foese, ${ }^{\mathrm{c}}$ Daniel Fulla-Marsa, ${ }^{\mathrm{a}}$ Sebastian Göde, ${ }^{\mathrm{a}}$ Mohammed Hassan, ${ }^{\text {b Jens Hauser, }}{ }^{\text {b }}$ Thomas Herrmannsdörfer, ${ }^{\text {b }}$ Hauke Höppner, ${ }^{\text {b }}$ Johannes Kaa, ${ }^{\text {e,a }}$ Peter Kaever, ${ }^{\text {b }}$ Klaus Knöfel, ${ }^{\text {b }}$ Zuzana Konôpková, ${ }^{a}$ Alejandro Laso García, ${ }^{\mathrm{b}}$ Hanns-Peter Liermann, ${ }^{\mathrm{c}}$ Jona Mainberger, ${ }^{\mathrm{c}}$

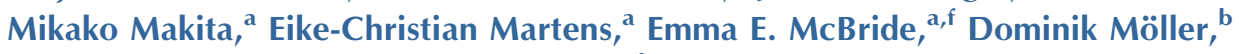
Motoaki Nakatsutsumi, ${ }^{a}$ Alexander Pelka, ${ }^{\mathrm{b}}$ Christian Plueckthun, ${ }^{\mathrm{a}}$ Clemens Prescher, ${ }^{\mathrm{c}}$ Thomas R. Preston, ${ }^{a}$ Michael Röper, ${ }^{\mathrm{c}}$ Andreas Schmidt, ${ }^{\mathrm{a}}$ Wolfgang Seidel, ${ }^{\mathrm{b}}$ Jan-Patrick Schwinkendorf, ${ }^{a}$ Markus O. Schoelmerich, ${ }^{a}$ Ulrich Schramm, ${ }^{\text {b Andreas Schropp, }}{ }^{\mathrm{c}}$ Cornelius Strohm, ${ }^{\mathrm{c}}$ Konstantin Sukharnikov, Peter Talkovski, ${ }^{c}$ Ian Thorpe, ${ }^{\mathrm{a}}$ Monika Toncian, ${ }^{\mathrm{b}}$ Toma Toncian, ${ }^{\mathrm{b}}$ Lennart Wollenweber, ${ }^{a}$ Shingo Yamamoto ${ }^{b}$ and Thomas Tschentscher ${ }^{a}$

${ }^{a}$ European XFEL, Holzkoppel 4, 22869 Schenefeld, Germany, ${ }^{\mathbf{b}}$ Helmholtz-Zentrum Dresden-Rossendorf eV, 01328 Dresden, Germany, ' Deutsches Elektronen-Synchrotron DESY, 22607 Hamburg, Germany, ${ }^{\mathbf{e}}$ Technische Universität Dortmund, 44227 Dortmund, Germany, and 'SLAC National Accelerator Laboratory, Menlo Park, CA 94025, USA. *Correspondence e-mail: ulf.zastrau@xfel.eu

The European XFEL delivers up to 27000 intense ( $>10^{12}$ photons) pulses per second, of ultrashort ( $\leq 50 \mathrm{fs}$ ) and transversely coherent $\mathrm{X}$-ray radiation, at a maximum repetition rate of $4.5 \mathrm{MHz}$. Its unique X-ray beam parameters enable groundbreaking experiments in matter at extreme conditions at the High Energy Density (HED) scientific instrument. The performance of the HED instrument during its first two years of operation, its scientific remit, as well as ongoing installations towards full operation are presented. Scientific goals of HED include the investigation of extreme states of matter created by intense laser pulses, diamond anvil cells, or pulsed magnets, and ultrafast X-ray methods that allow their diagnosis using self-amplified spontaneous emission between 5 and $25 \mathrm{keV}$, coupled with X-ray monochromators and optional seeded beam operation. The HED instrument provides two target chambers, X-ray spectrometers for emission and scattering, $\mathrm{X}$-ray detectors, and a timing tool to correct for residual timing jitter between laser and X-ray pulses.

\section{Scientific scope}

The High Energy Density (HED) scientific instrument at the European X-ray Free-Electron Laser Facility GmbH (European XFEL) (Tschentscher et al., 2017; Decking et al., 2020) is a unique platform for experiments combining hard $\mathrm{X}$-ray free-electron laser radiation with the capability to generate matter at extreme conditions of pressure, temperature, or electromagnetic fields. The instrument started user operation in May 2019 and will augment its capabilities within the next years.

The scientific aims of the HED instrument are summarized in the HED Conceptual Design Report (CDR) (Nakatsutsumi \& Tschentscher, 2013). In general, HED science sheds light 
on properties of matter with an energy density exceeding $10^{11} \mathrm{~J} \mathrm{~m}^{-3}$, which is equivalent to a pressure of $>1 \mathrm{Mbar}$, a magnetic field strength of $500 \mathrm{~T}$, or the internal energy of a hydrogen atom (Drake, 2010). These conditions prevail, for example, in planetary and stellar interiors, impact scenarios, and in intense laser-matter interactions in both fundamental research and industrial applications.

Located downstream of the SASE2 undulator, which provides photon energies of $5-25 \mathrm{keV}$, the HED instrument focuses on experiments which require hard X-rays, allowing volumetric studies of high-density material, pumping certain atomic resonances or covering a large reciprocal space in crystallographic structure analysis. In particular, the instrument is optimized to exploit the pulse structure of the European XFEL, offering an up to $600 \mu$ s-long train of X-ray pulses each separated by $222 \mathrm{~ns}$ [equivalent to a rate of $(222 \mathrm{~ns})^{-1}=4.514 \mathrm{MHz}$, at a base repetition rate of $10 \mathrm{~Hz}$, therefore delivering a bright X-ray burst every $100 \mathrm{~ms}$. Single or multiple pulses in a train at $10 \mathrm{~Hz}$ or on demand can be selected. The provided instrumentation is tailored to match this $10 \mathrm{~Hz}$ repetition rate and to make use of the unique pulse train structure. In comparison with conventional HED platforms, this increases the data rate and hence leads to a paradigm shift regarding statistics and the ability to explore a wider parameter space during a single experimental campaign. The unique $\mathrm{MHz}$ capability means that the evolution of submillisecond dynamics can be recorded with a single X-ray burst. This is particularly interesting for processes which do not permit accumulative measurements or cannot be repeated frequently (Cerantola et al., 2021). Key scientific cases for the HED instrument are summarized below.

At gigapascal pressures, complex crystallographic and chemical states of matter may form (Appel et al., 2014). At the HED instrument, static high-pressure conditions of several $100 \mathrm{GPa}$ can be generated using diamond anvil cells (DACs). The pressure can be dynamically varied using DACs equipped with piezoactuators (dynamic DAC, dDAC) (Jenei et al., 2019) and the temperatures increased via absorption of X-rays (Meza-Galvez et al., 2020) or infrared lasers (Konôpková et al., 2016).

Studying the response of materials to different strain rates allows to differentiate between plastic and elastic processes and study the kinetics of phase transitions. Dynamic loading at even higher strain rates and to higher pressures can be studied in shock wave experiments using laser ablation pressure. A diode-pumped laser (De Vido et al., 2019) with 2-15 ns pulse duration at $10 \mathrm{~Hz}$ (Section 5.3.2) providing up to $100 \mathrm{~J}$ in the infrared will enable the generation of transient pressures of up to $1 \mathrm{TPa}$ (Zastrau et al., 2017). Such ultrahigh pressures lead to discoveries of unique physical and chemical phenomena and a deeper understanding of matter.

A combination of a femtosecond (fs) duration, high-intensity optical laser with an XFEL allows to study transient, nonequilibrium states on ultrafast time scales (Nakatsutsumi et al., 2016). This allows the study of the electron-ion thermalization dynamics, non-thermal melting and associated transport properties under a priori known ion density, to name a few.
Understanding these processes provides deeper insight into material processing via ablation and warm dense matter (WDM) - a dense plasma state between the ideal solid and an ideal plasma. At electron-relativistic laser intensity $\left(\geq 10^{18} \mathrm{~W} \mathrm{~cm}^{-2}\right)$, generation of the extremely high magnetic fields (Wang et al., 2019), growth rates of instabilities (Ruyer et al., 2020; Göde et al., 2017) and collisionless shocks (Fiuza et al., 2020) can be investigated. Fundamental understanding of these transient phenomena also helps to optimize and control the generation of bright particles (Wilks et al., 2001), X-ray line emission (Zastrau et al., 2010) and coherent attosecond extreme ultraviolet pulses (Wheeler et al., 2012) as a secondary source for a probe or a driver.

Instead of using an optical laser which interacts with only the sample surface, the volumetric formation of WDM using intense X-rays has shown great potential over the last decade (Vinko et al., 2012; Sperling et al., 2015.). Studies can be readily extended to X-ray pump/X-ray probe techniques using a splitand-delay line (Lu et al., 2018), as well as two-color operation of the XFEL itself.

A pulsed magnetic field setup allows the stabilization of otherwise inaccessible new states of matter. Of interest are correlated electron systems where spin, orbital, charge and lattice degrees of freedom act on similar energy scales and lead to competing ground states. One of these so far unknown phases is the state of high- $T_{\mathrm{c}}$ superconductors above the critical magnetic field, when the superconducting property has collapsed (Grissonnanche et al., 2016). Other systems of current interest are frustrated magnets from triangular lattices that show rich phase diagrams at high magnetic fields (Wosnitza et al., 2016), and materials showing topological order (Liu et al., 2017).

\section{The HIBEF user consortium}

The Helmholtz International Beamline for Extreme Fields (HIBEF) user consortium (UC) contributes instrumentation and operation staff to the HED instrument. HIBEF is led by the Helmholtz Zentrum Dresden-Rossendorf (HZDR), and other major contributors are the Deutsches ElektronenSynchrotron (DESY) and the UK's Scientific and Technology Facilities Council (STFC). The HIBEF-provided instrumentation represents an integral part of the HED instrument - major contributions of the UC include high-intensity and high-energy laser systems (see Sections 5.3.1 and 5.3.2), a dedicated interaction chamber for precision X-ray diffraction (XRD) studies (see Section 6.4) in laser-shock and DAC experiments, and a setup for studying samples at cryogenic temperatures in pulsed magnetic fields (see Sections 5.4 and 6.5). While the European XFEL is responsible for the provision of beam time, staff from HIBEF and the European XFEL operate, maintain and develop the experimental platforms jointly. European XFEL publishes an open call for proposals typically twice a year in May and November. 


\section{Technical infrastructure}

\subsection{European XFEL and the SASE2 undulator source}

The European XFEL is a free-electron laser (FEL) user facility currently delivering soft and hard X-ray FEL radiation to six scientific instruments (Tschentscher et al., 2017). It provides X-rays with high average brilliance and extreme peak intensities at photon energies up to $25 \mathrm{keV}$ in the fundamental, with femtosecond pulse duration and a high degree of coherence. The high average brilliance is achieved through acceleration of up to 2700 electron bunches with typically $0.25 \mathrm{nC}$ at a $10 \mathrm{~Hz}$ repetition rate by a superconducting electron accelerator (Weise \& Decking, 2017) (Fig. 1). After reaching energies of $11.5,14$, or $16.5 \mathrm{GeV}$, these electrons are distributed to three beamlines housing self-amplified spontaneous emission (SASE) undulators (Abeghyan et al., 2019) with horizontal polarization. Tuning of the photon energy is achieved independently in each beamline by adjusting the undulator gaps. The HED science instrument is located after the SASE2 undulator which produces short $\sim 25$ fs X-ray pulses with photon energies from 5 to $25 \mathrm{keV}$ (Scholz \& Zhao, 2019).

The highest achieved SASE2 levels are summarized in Fig. 2 - average performance is at $50-80 \%$ of these values. Typically, a SASE spectrum has a fully tunable Gaussian spectral envelope with bandwidth $\Delta E / E \simeq 10^{-3}$. Within this bandwidth, the spectrum consists of several longitudinal modes leading to $<1 \mathrm{eV}$ broad spikes which statistically fluctuate from pulse to pulse. Example spectra are shown in Section 4.2.2. SASE2 can also operate in hard X-ray self-seeding mode (HXRSS) (Geloni et al., 2011, 2019; Liu et al., 2019), producing a more coherent and narrow-bandwidth pulse. Current studies performed at 7.5 and $9 \mathrm{keV}$ photon energy yield pulse energies of $\sim 1 \mathrm{~mJ}$ in a spectral bandwidth of $\sim 1 \mathrm{eV}$.

The initial beam divergence from the undulator can be measured using imagers along the beam transport, which in turn allows to estimate the diffraction-limited source size as a function of photon energy, as shown in Table 1.

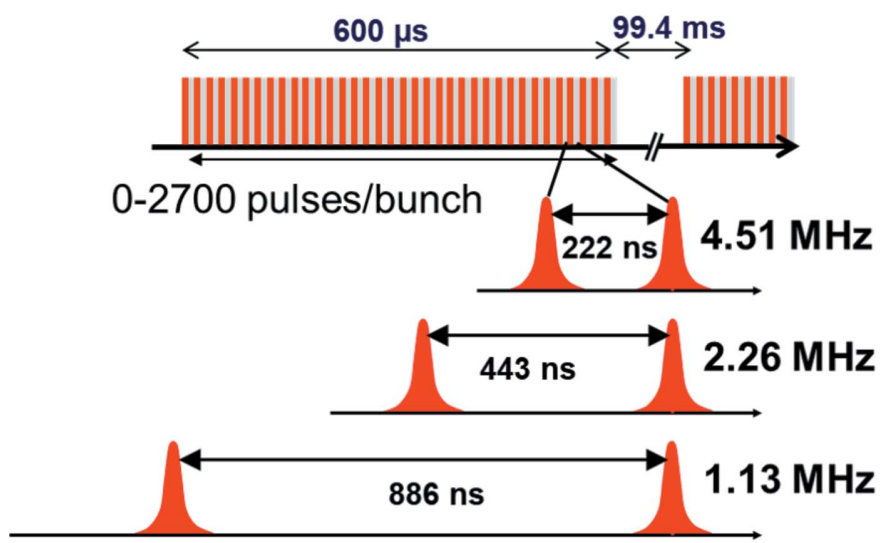

Figure 1

Unique bunch pattern of European XFEL. Within an up to $600 \mu$ s-long window, between a single and $2700 \mathrm{X}$-ray pulses can be created. The facility can deliver pulses at difference repetition rates of 4.51, 2.26, or $1.13 \mathrm{MHz}$, resulting in a pulse spacing of 222, 443 or $886 \mathrm{~ns}$, respectively.
Table 1

SASE2 undulator divergence (measured with imagers) and source size (calculated from divergence) for $0.25 \mathrm{nC}$ bunch charge.

\begin{tabular}{llll}
\hline $\begin{array}{l}\text { Linac electron } \\
\text { energy }(\mathrm{GeV})\end{array}$ & $\begin{array}{l}\text { SASE photon } \\
\text { energy }(\mathrm{keV})\end{array}$ & $\begin{array}{l}\text { Source divergence } \\
(\mu \mathrm{rad})\end{array}$ & $\begin{array}{l}\text { Source } \\
\text { size }(\mu \mathrm{m})\end{array}$ \\
\hline 11.5 & 6.0 & $2.44 \pm 0.25$ & $\sim 43$ \\
14.0 & 9.0 & $2.33 \pm 0.25$ & $\sim 30$ \\
16.5 & 17.8 & $1.68 \pm 0.25$ & $\sim 21$ \\
\hline
\end{tabular}

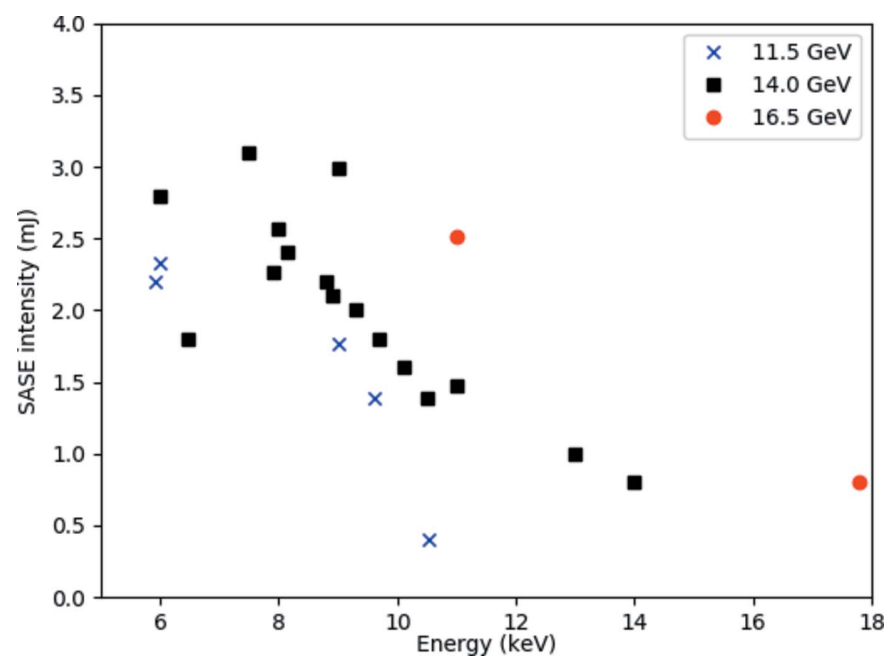

Figure 2

Current peak performance of the SASE2 FEL at photon energies between 6 and $18 \mathrm{keV}$. The data points represent peak pulse energies measured in 2019 and 2020 (https://xfel.desy.de/operation/performance/; see also Maltezopoulos et al., 2019). The linac was operated at $11.5 \mathrm{GeV}$ (blue crosses), $14 \mathrm{GeV}$ (black rectangles) and $16.5 \mathrm{GeV}$ (red circles) electron energy.

\subsection{HED instrument layout}

The HED instrument is located in the experiment hall at the end of a $\sim 970$ m-long photon beam transport tunnel partially shared with the Materials and Imaging Dynamics (MID) instrument (Madsen et al., 2021). The basic layout of the HED hutches is described in the Technical Design Report (TDR) (Nakatsutsumi et al., 2014) and the layout of the optics and experiment hutch is shown in Fig. 3.

The experiment hutch and the optics hutch are air-conditioned and H14-filtered and kept at a temperature of $21 \pm 1^{\circ} \mathrm{C}$ and a humidity of $50 \pm 2.5 \%$. Dedicated zones exist with precision temperature control: $21 \pm 0.1^{\circ} \mathrm{C}$. Details of the experiment hutch arrangement are described in Section 6. On the roof of the experiment hutch is a laser bay which houses the two large laser systems ReLaX and DiPOLE (see Fig. 4 and Sections 5.3.1 and 5.3.2) in a clean-room-like laboratory with precision temperature $\left(21 \pm 0.1^{\circ} \mathrm{C}\right)$ control. Adjacent to this are rack rooms where the majority of the control electronics and power supplies are placed.

\subsection{Control systems}

The European XFEL's Python-based software framework for control and data acquisition is named Karabo (Heisen et al., 2013). It allows the user to build extendable distributed 


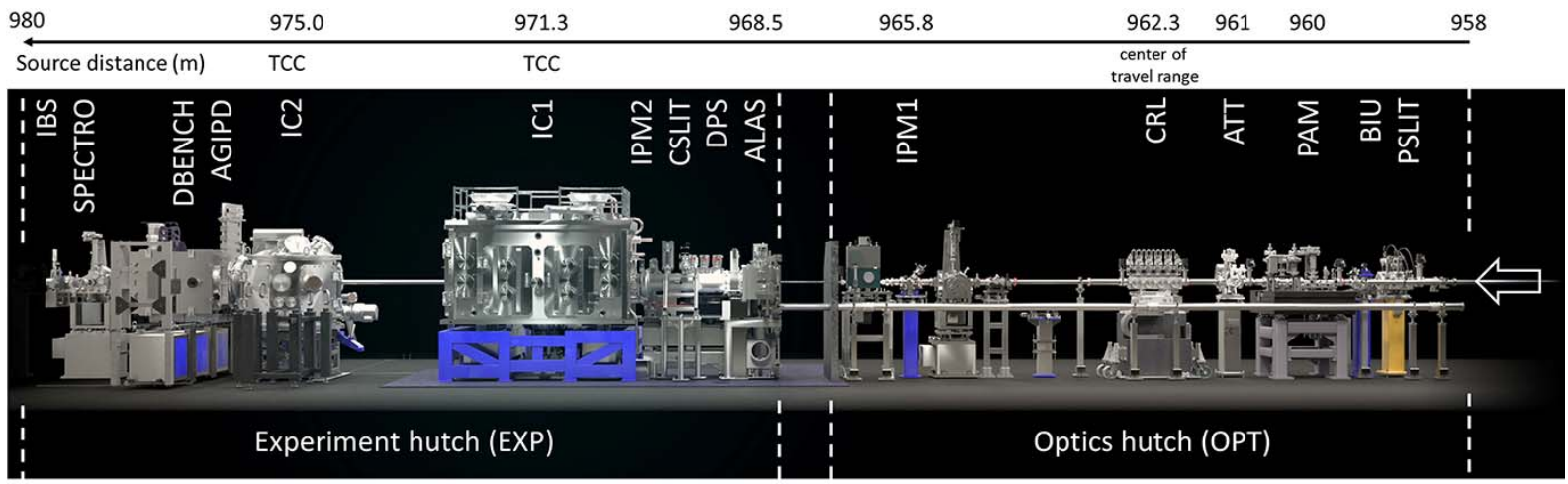

Figure 3

HED optics and experiment hutch layout. The X-rays enter from the right. Optics hutch: PSLIT - water-cooled four-blade power slits, BIU - beam imaging unit, PAM - photon arrival monitor for X-ray-optical laser timing, ATT - solid attenuator foils, CRL - compound refractive lenses made of Be (CRL3), IPM1 - intensity and position monitor. Experimental hutch: ALAS: incoupling for alignment laser, DPS - differential pumping, CSLIT - cleanup slits, two four-blade assemblies for soft and hard X-rays, respectively, IPM2 - intensity and position monitor, IC1 and IC2 - interaction chambers, AGIPD - MHz repetition compatible X-ray detector, DBENCH - detector bench, SPECTRO - position of downstream spectrometers, IBS - instrument beam stop. The distances on the top are given from the source (center of last undulator segment).

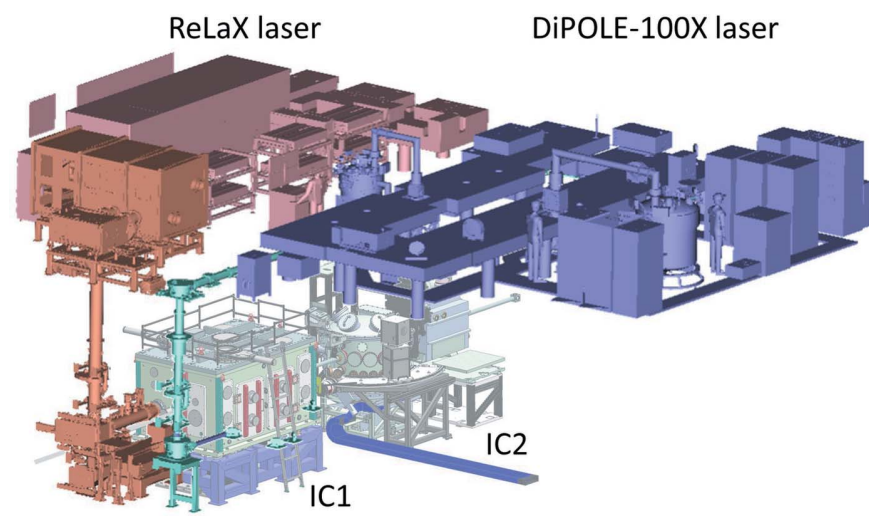

Figure 4

Schematics showing the position of the ReLaX and DiPOLE 100-X laser in a room above the experiment hutch with the interaction chambers IC1,2 (walls not shown).

applications that can be remotely controlled through a graphical user interface (GUI) or command line interface. While acting as a control system, Karabo interfaces with hardware controllers via driver devices. The communication between the Karabo devices as well as instrument- and experiment-specific programming can be implemented in socalled Karabo middle layer devices and macros (Hauf et al., 2019).

The control hardware can be divided into two categories: slow real-time control (motion control, vacuum system, water flow and temperature control) and fast electronics for timing and analog-digital conversion. The slow real-time control is managed by an industrial standard Programmable Logic Controller (PLC) system based on EtherCAT bus terminals and CPUs from Beckhoff (Gessler et al., 2020) enhanced by multi-channel-Piezo motion controllers with interlock capability that are directly interfaced to the Karabo control system.

The use of the industrial PLC system facilitates standardization and modularization of software and hardware. It also allows the implementation of equipment protection interlocks, motorized axis coupling, and feedback control. The fast electronics platform uses MicroTCA crates for timing, digitization of analog signals, and online signal processing (Gessler et al., 2020) which matches the pulse rate of European XFEL.

The timing precision performance of the Karabo-controlled software layer is typically well below the $100 \mathrm{~ms}$, but high network traffic can cause latency. Therefore, for $10 \mathrm{~Hz}$ pulseto-pulse actions such as triggering an acquisition, the real-time PLCs are preferred. Sampling of data within the pulse train with 222 ns intervals, or even resolving sub-ns pulse shapes, is possible using the the MicroTCA with sampling rates of up to 0.5 Gigasamples $\mathrm{s}^{-1}$.

\section{X-ray transport, optics and diagnostics}

A schematic overview of the $\sim 1 \mathrm{~km}$-long X-ray beam transport from the SASE2 undulator to the HED instrument beam stop is given in Fig. 5. For the first 390 m, the HED and MID instruments share a common beam transport. The first optics are a set of interchangeable lenses (CRL1) that allow the beam to be collimated or focused directly to the sample position (Nakatsutsumi \& Tschentscher, 2013; Nakatsutsumi et al., 2014). At photon energies of $6 \mathrm{keV}$, the beam size at CRL1 is just below $1 \mathrm{~mm}$ full width at half-maximum (FWHM) in accordance with design parameters (Schneidmiller \& Yurkov, 2011). Next comes a pair of horizontal deflecting mirrors (M1, M2), with a usable length of $80 \mathrm{~cm}$, which horizontally offset the beam in order to suppress high harmonics as well as bremsstrahlung and spontaneous synchrotron radiation.

Higher harmonics are weak multiples of the fundamental photon energy. While odd harmonics are created in every undulator on-axis, even ones are found in a cone slightly offaxis. With chromatic focusing optics (such as beryllium lenses) they are not focused to the same spot as the fundamental. In particular, the strong third harmonic often disturbs diffraction 


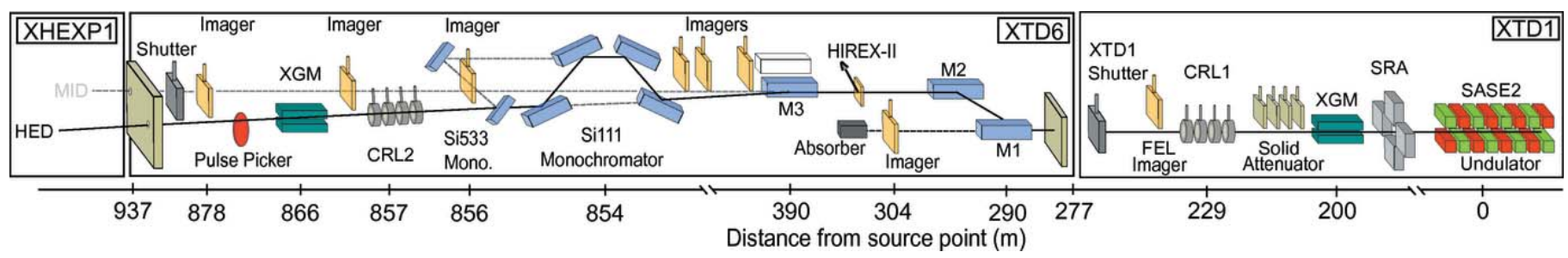

Figure 5

Beam transport in the XTD1 and XTD6 underground tunnels up to the XFEL Headquarters EXPeriment hall 1 (XHEXP1). The X-rays enter from the right. Not all components are shown. A detailed list of devices and distances can be found in Appendix $B$.

patters and creates X-ray fluorescence. On the other hand, it can be used as hard X-ray source in excess of the $25 \mathrm{keV}$ limit of the fundamental.

The grazing-incidence angle of M1 and M2 can be varied between 1.7 and $3.6 \mathrm{mrad}$ and the mirrors have both $\mathrm{B}_{4} \mathrm{C}$ and $\mathrm{Pt}$ coatings for low and high photon energy operation, respectively, to ensure high reflectivity across the entire photon energy range. The reflectivity is shown in Fig. 6. A third, mechanically identical mirror (M3) can be inserted at $390 \mathrm{~m}$ with a fixed incidence angle of $1.3 \mathrm{mrad}$ to steer the beam to the HED instrument (Tschentscher et al., 2017; Sinn et al., 2019).

Even in the case of a collimated beam, overfilling of the M3 mirror can occur at mid-to-low photon energy $(\leq 15 \mathrm{keV})$ (Nakatsutsumi \& Tschentscher, 2013). To overcome this problem, an intermediate focusing scheme before the downstream optics using the CRL1 can be applied. In addition, M2 is bendable which allows both the correction of the horizontal beam size and potential astigmatism from thermal mirror deformation (Vannoni et al., 2019). The transmission of the beamline over the mirrors is typically $\sim 70-80 \%$.

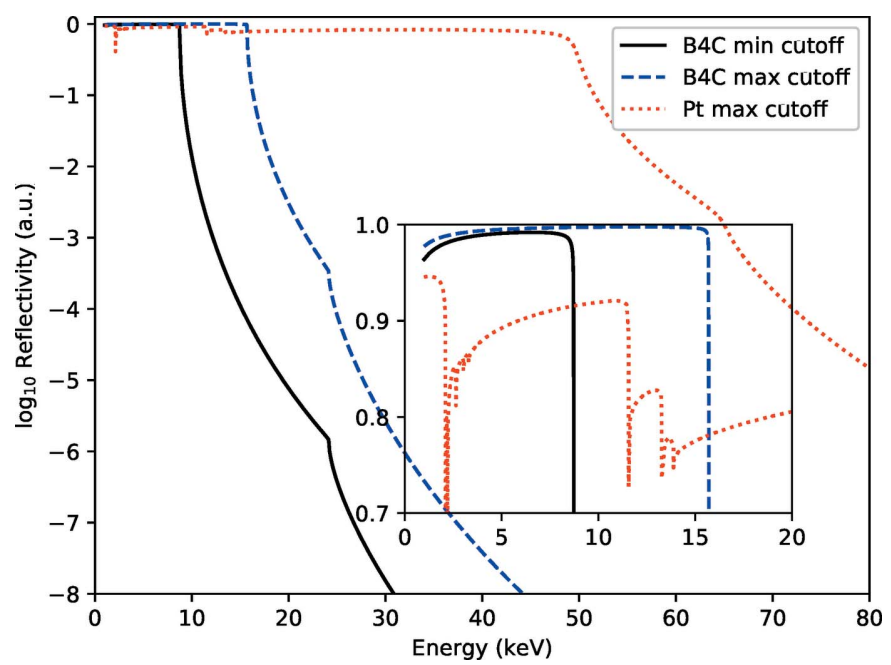

Figure 6

Calculated total external reflectivity of the combined mirrors M1-M3 for both reflecting coatings plotted on a log scale, inset on linear scale. Shown is the minimum energy cut-off using the $\mathrm{B}_{4} \mathrm{C}$ coating for all three mirrors and mirrors $\mathrm{M} 1$ and $\mathrm{M} 2$ at $3.6 \mathrm{mrad}$ suitable to reduce third harmonic content, and the maximum energy cut-off using the Pt coating for all three mirrors and mirrors $\mathrm{M} 1$ and $\mathrm{M} 2$ at $1.7 \mathrm{mrad}$. Also shown is the typically used working point at $2 \mathrm{mrad}$ using the $\mathrm{B}_{4} \mathrm{C}$ coating. In all cases the mirror M3 is set to its working point of $1.3 \mathrm{mrad}$.
The vertical divergence of the X-ray beam can be adjusted to match horizontal divergence by bending the M2 mirror. The resulting beam has a circular shape; however, beam jitter and weak diffraction from the M1-M3 mirror edges result in a slight horizontally broadening.

Changes in the beam pointing from the undulator, or thermal drift of mirrors M1-M3, can lead to large beam drifts after $\sim 1 \mathrm{~km}$ of photon beam transport. Therefore an active beam stabilization system has been implemented. The X-ray beam pointing is constantly monitored by highly transmissive boron-doped chemical vapor deposition (CVD) diamond screens downstream of the mirrors. A feedback loop acts on piezo actuators on mirrors M2 and M3 to correct the beam pointing.

\subsection{X-ray focusing optics}

The X-ray focusing is based on Be CRLs (Lengeler et al., 1999). These optics are chromatic, which requires different lens configurations for each photon energy (Zozulya et al., 2019; Nakatsutsumi et al., 2014).

Three groups of lenses are placed at different positions along the HED beamline (labeled CRL1-3) in order to ensure the maximum throughput of photons by matching the aperture to the FEL beam size. Several focal schemes and the lens configurations are shown in Appendix B. Depending on photon energy and not taking into account the chromatic aberration due to the finite SASE bandwidth, the smallest FWHM beam sizes that can be theoretically achieved at the target chamber center (TCC) are 150-250 $\mu \mathrm{m}$ with CRL1, $\sim 15 \mu \mathrm{m}$ with CRL2 and $1-2 \mu \mathrm{m}$ with CRL3. The CRL2 is placed after the monochromators in order to keep the divergence over the monochromators low.

Since the lenses are chromatic, a SASE spectrum will be focused across a finite range. Table 2 compares the Rayleigh length of the monochromatic beam with the actual focal length spread according to $0.3 \%$ FWHM spectral bandwidth using an intermediate focus with CRL1 and subsequent tight focusing with CRL3. The entire chamber of CRL3 can be translated by $\pm 50 \mathrm{~cm}$ along the beam axis to overlap focal positions and TCC.

In order to characterize the shape and size of the X-ray focus, single-pulse imprints in lithium fluoride (LiF) creating permanent point-defects (Pikuz et al., 2015) were taken and yield a focal spot of 4-5 $\mu \mathrm{m}$ FWHM at $6 \mathrm{keV}$ with SASE beam 
Table 2

Calculation of the Rayleigh length of the monochromatic beam and focal spread which includes a $0.3 \%$ FWHM spectral bandwidth using CRL1 intermediate focus and CRL3 tight focus.

Note that a diffraction-limited focus can only be achieved up to $20.5 \mathrm{keV}$ at the TCC of IC1 - beyond that the refractive power of the installed lenses is not sufficient. Therefore, a pre-focusing with CRL1 combined with CRL3 focusing at the IC2 chamber is shown. A tight focus above $20.5 \mathrm{keV}$ is possible by replacing some weaker lenses.

\begin{tabular}{llll}
\hline $\begin{array}{l}\text { SASE photon } \\
\text { energy }(\mathrm{keV})\end{array}$ & $\begin{array}{l}\text { Diffraction } \\
\text { limit focus } \\
(\mu \mathrm{m})\end{array}$ & $\begin{array}{l}\text { Rayleigh length of } \\
\text { monochromatic } \\
\text { beam }(\mathrm{mm})\end{array}$ & $\begin{array}{l}\text { Rayleigh length } \\
\text { with 0.3\% } \\
\text { bandwidth }(\mathrm{mm})\end{array}$ \\
\hline 6.0 & 1.0 & 4.1 & 28.5 \\
8.0 & 0.8 & 3.0 & 28.5 \\
9.0 & 0.8 & 3.8 & 29.5 \\
13.0 & 0.3 & 0.9 & 27.5 \\
18.0 & 0.3 & 1.0 & 28.2 \\
25.0 & 0.7 & 8.3 & 43.6 \\
\hline
\end{tabular}

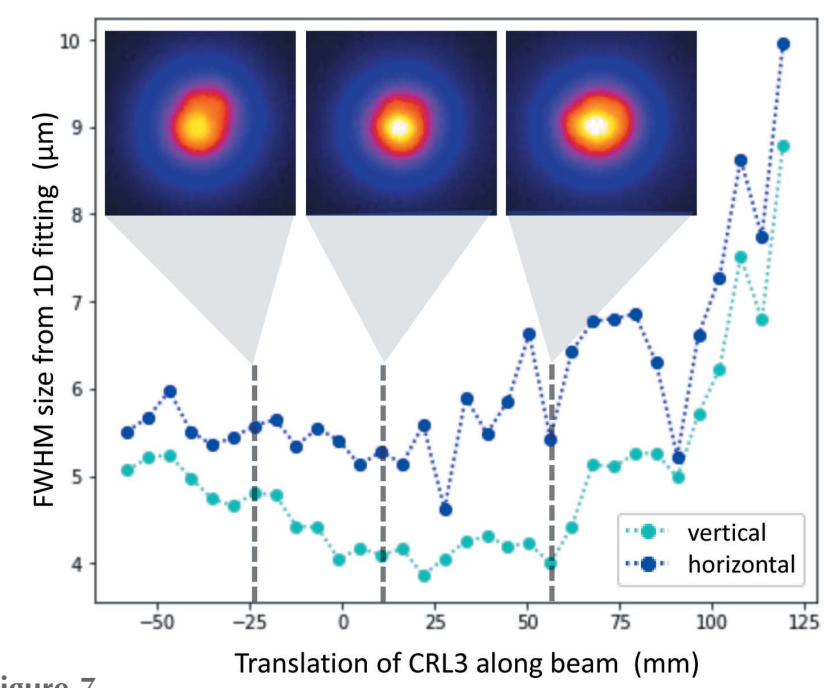

Figure 7

Characterization of the best focus of CRL3 and an intermediate focus scheme with CRL1 at $6 \mathrm{keV}$ photon energy. The three false-color insets are single-shot imprints into a $\mathrm{LiF}$ crystal at $0.5 \%$ beamline transmission.

(Fig. 7). Due to chromatic aberration, the Rayleigh range extends over several tens of $\mathrm{mm}$.

Alternatively, scanning methods with an obstacle project the focal size along the scan direction. Figure 8 shows the results of scanning the round edge of a $1 \mathrm{~mm}$-diameter tungsten wire across the focused $\mathrm{X}$-rays at $17.8 \mathrm{keV}$, resulting in a $4.5 \mu \mathrm{m} \times 5 \mu \mathrm{m}$ spot $(\mathrm{FWHM})$. While providing immediate focal size estimates, the result is potentially broadened by beam pointing jitter.

In order to reach X-ray foci smaller than $1 \mu \mathrm{m}$, both interaction chambers can be equipped with CRLs with adjustable short-focal length between about $10 \mathrm{~cm}$ and $1 \mathrm{~m}$. This nano-focusing capability (CRL4) is contributed by the HIBEF UC. These lenses have radii of curvature of $50 \mu \mathrm{m}$, an effective aperture between $300 \mu \mathrm{m}$ and $400 \mu \mathrm{m}$, and up to 50 of them can be hosted in a lens cassette, similar to the concept described by Schropp et al. (2012). Slight pre-focusing is necessary to match the aperture and achieve maximum throughput.
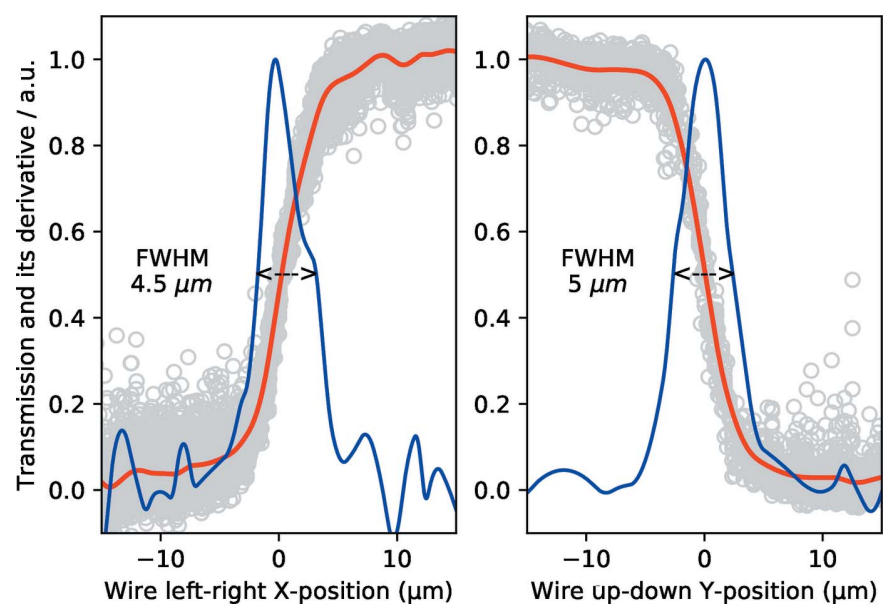

Figure 8

Characterization of the best focus of CRL3 after intermediate focus with CRL1 at $17.8 \mathrm{keV}$ with a wire scan. The scans took a few minutes with $5 \%$ transmission. The $y$-axis shows in red the transmission (normalized to the incident pulse energy, and with a moving average), as well as its derivative in blue.

A focus spot size of $\sim 220 \mathrm{~nm}$ at the TCC of IC1 has been demonstrated using a SASE beam at $9 \mathrm{keV}$ photon energy. The average focus size was retrieved from scanning ptychography (Schropp et al., 2013) and yields an upper limit of $220 \mathrm{~nm}$ FWHM for a monochromatic beam and $\leq 300 \mathrm{~nm}$ FWHM for SASE. For the SASE case, we could not resolve a difference between a ten-shot average and a single exposure, indicating a focal jitter of less than $\sim 500 \mathrm{~nm}$.

\subsection{X-ray monitors and diagnostics}

4.2.1. 2D imagers. Several 2D beam imaging monitors are installed throughout the optical path. The monitors are based on fluorescent screens such as boron-doped diamond or single-crystal cerium-doped yttrium aluminium garnet (YAG:Ce) that can be inserted at an angle to the XFEL beam (Koch et al., 2019; Grünert et al., 2019). The visible-light fluorescence of the beam is then recorded on a $10 \mathrm{~Hz}$ frame-rate camera that is positioned at an angle of $90^{\circ}$ to the X-ray beam. All imagers and their properties are listed in Appendix $B$. Since not all imagers are permanently inserted into the beam path, recording of their data to the data acquisition system has to be actively enabled for each imager.

4.2.2. Bent crystal spectrometers. The stochastic nature of the SASE process results in spectral fluctuations from pulse to pulse. In order to record a single pulse-resolved spectrum, the use of cylindrically bent $\mathrm{Si}$ crystals has been practiced widely (Zhu et al., 2012) as a transmissive Bragg spectrometer, albeit its application is limited to a few tens of pulses at $\mathrm{MHz}$ repetition rates. A recent development using cylindrically bent diamond crystals has shown comparable spectral resolution (Boesenberg et al., 2017; Samoylova et al., 2019) and radiation tolerance for full $4.5 \mathrm{MHz}$ pulse trains at $9 \mathrm{keV}$ photon energy. There are three opportunities for inserting such spectrometers into the beam path, all of them equipped with both diamond and $\mathrm{Si}$ crystals with various bending radii and cuts. 
Upstream of the sample, the HIREX-II (Kujala et al., 2020) spectrometer is permanently installed between mirrors M2 and M3. It covers the photon energy range $5-25 \mathrm{keV}$ at a resolution of $\leq 0.2 \mathrm{eV}$. The reflected signal can either be recorded with a $10 \mathrm{~Hz} 2 \mathrm{D}$ sCMOS detector, or at $4.5 \mathrm{MHz}$ with a 1D X-ray GOTTHARD detector (Mozzanica et al., 2012). For experiments that require the spectral information right up- or downstream of a sample, additional bent-crystal spectrometers HED-flex and CNRS-spec can be placed simultaneously. The CNRS-spec [contribution from Center National de la Recherche Scientifique (CNRS), France] can flexibly be placed inside or outside the IC1 chamber, while the HED-flex can be placed in-air downstream of IC1. Both the HED- and CNRS-spec are designed to be inserted directly in the beam, similar to the HIREX-II spectrometer. Both are also coupled with either a $10 \mathrm{~Hz} 2 \mathrm{D}$ optical camera or a 1D GOTTHARD X-ray detector. The HED-flex spectrometer has been tested at $6 \mathrm{keV}$ in the diverging beam (see Fig. 9) using $\mathrm{Si}(111)$ crystal with $78 \mathrm{~mm}$ bending radius, and a $10 \mathrm{~Hz}$ ANDOR sCMOS ZYLA 5.5 camera, imaging a $25 \mu \mathrm{m}$ YAG:Ce screen, with a magnifying microscope objective. The minimum energy resolution for this arrangement was $0.13 \mathrm{eV}$.

A detailed description of the spectrometer configurations can be found in Appendix $B$.

4.2.3. Intensity and position monitors. X-ray gas monitors (XGMs) for non-invasive single-shot pulse energy measurements and average beam postion monitoring are installed at two positions in the HED beamline (Grünert et al., 2019; Maltezopoulos et al., 2019; Sorokin et al., 2019). The first is placed in the XTD1 tunnel, upstream of all optics and attenuators to measure the output of the undulator. The second XGM is installed in the HED branch in the XTD6 tunnel after all the main optics. Details on their operation are described by Maltezopoulos et al. (2019).

Two compact intensity and position monitors (IPMs) are installed at the HED instrument. The IPM in the HED optics hutch is positioned downstream of the high-power slit system, solid attenuators and CRL3 and therefore allows the transmission of these devices to be measured. The second IPM in the experiment hutch is installed downstream of the differ-
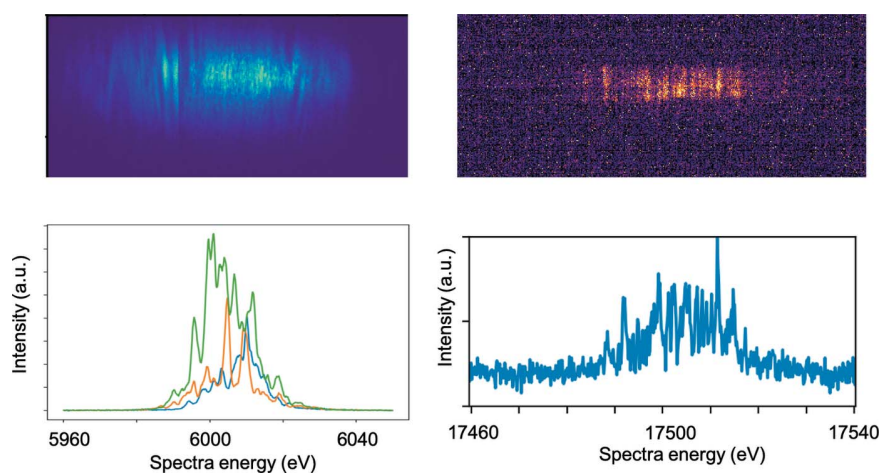

Figure 9

Examples of single-pulse spectra. Spectra as recorded by a 2D detector imaging a scintillator screen (top) and their corresponding lineouts (bottom). Left: HED-flex at $6 \mathrm{keV}$ using a $\mathrm{Si}(111)$ crystal and lineouts of three different pulses to illustrate the shot-to-shot fluctuation. Right: HIREX-II in the XTD6 tunnel at $17.5 \mathrm{keV}$ using C(110). ential pumping system and the clean-up slits. Both devices insert a thin diamond screen into the beam path and record the backscattering on four diodes (Hamamatsu S3590-09), providing pulse-resolved intensities and positions at $4.5 \mathrm{MHz}$. The signal difference between the up-down and left-right diode pairs yields the beam position. For absolute intensity measurements, the IPMs require cross-calibration to the XGMs for each specific photon energy. Since the intensity signal depends on the thickness of the screen, they have less than $2 \%$ thickness variation across the central $5 \mathrm{~mm}$, and less than $5 \%$ over the entire area. Several thicknesses of CVD diamond screens are available; currently these are $22 \mu \mathrm{m}$, $50 \mu \mathrm{m}$ and $100 \mu \mathrm{m}$. Furthermore, the instrument beam stop (IBS) also incorporates a fast diode monitoring the X-ray scattering from a permanently installed $500 \mu \mathrm{m}$-thick CVD diamond screen.

\subsection{Monochromators}

An Si(111) four-bounce monochromator (Dong et al., 2016) consisting of two pairs of artificial channel-cut crystals can be inserted into the beam path. It reduces the bandwidth to $\Delta E / E \simeq 1 \times 10^{-4}$ (Fig. 10) for the complete range of photon energies. Both pairs of crystals can be cryogenically cooled to mitigate the heat load during a pulse train. The second pair of the $\mathrm{Si}(111)$-monochromator can be replaced with a $\mathrm{Si}(533)$ channel-cut crystal, yielding a bandwidth of $\Delta E / E \simeq 4 \times 10^{-6}$ at a fixed photon energy of $7494 \mathrm{eV}$. More details are given by Wollenweber et al. (2021). Both configurations provide zero offset between the incident and the monochromated beam. In order to keep the beam divergence on the crystals small, the monochromators are positioned upstream of the CRL2 and CRL3 optics. The beam jitter after the cryogenically cooled $\mathrm{Si}(111)$ monochromator was measured by the imager screen at the end of the XTD6 tunnel, $22 \mathrm{~m}$ downstream of the monochromator, to be $\sim 40 \mu \mathrm{m}$ in both horizontal and vertical.

\subsection{Slit systems}

The HED instrument includes three sets of slit systems to control the size of the beam and its potential halo. Such a halo can be caused by scattering from upstream slits, apertures,

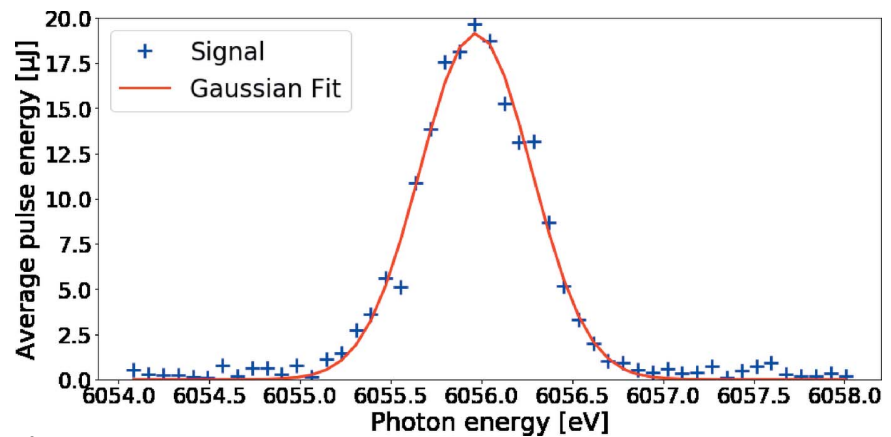

Figure 10

Measured energy resolution of the $\mathrm{Si}(111)$ monochromator at $E=$ $6055 \mathrm{eV}$. The transmitted SASE X-ray pulse energy of the four-bounce setup is shown as a function of the Bragg angle (scaled to photon energy) of the second pair of crystals while the first pair was fixed. The Gaussian fit yields a FWHM of $\Delta E=0.72 \mathrm{eV}$ and $\Delta E / E \simeq 1.2 \times 10^{-4}$. 
screens or filters. The water-cooled power slits are the first device located in the optics hutch, $\sim 15 \mathrm{~m}$ upstream of the TCC in IC1, and this system comprises four independent, nonintersecting slits with travel ranges of $\pm 25 \mathrm{~mm}$. The slits are $5 \mathrm{~mm}$ blades of tungsten carbide bonded to an absorbing block of $70 \mathrm{~mm}$-thick $\mathrm{B}_{4} \mathrm{C}$. The blades have highly polished knifeedges with a slope of $0.5^{\circ}$ and can be positioned with an accuracy of $0.4 \mu \mathrm{m}$.

In the experiment hutch $\sim 2 \mathrm{~m}$ before the TCC of IC1, there are two sets of clean-up slits, consisting of non-intersecting blades with travel ranges of $\pm 15 \mathrm{~mm}$. These can clean up potential scattering introduced by the first slit system and the CRLs. The first pair is similar to the power slit: it uses $4 \mathrm{~mm}$ tungsten carbide blades, with a highly polished $0.5^{\circ} \mathrm{knife-edge,}$ mounted to $6 \mathrm{~mm}$-thick $\mathrm{B}_{4} \mathrm{C}$ absorbers. The second pair, however, provides a round edge made from $\mathrm{Si}_{3} \mathrm{~N}_{4}$ and mounted to a $3 \mathrm{~mm}$ tantalum blade. These slits can also be positioned with an accuracy of $<1 \mu \mathrm{m}$.

The clean-up slits can also be used to decrease the content of higher harmonics in the beam, which is typically less focused near the TCC due to the chromaticity of the CRLs.

\subsection{Attenuators and pulse picker}

Solid attenuators are placed in the HED beamline at two positions. The upstream attenuator is positioned in the common SASE2 branch, downstream of the first XGM and upstream of the beamline optics (see Fig. 5). It comprises six chemical vapor deposition (CVD) diamonds $(0.075-2.4 \mathrm{~mm}$ thick) and three Si filters (0.5-2.0 mm thick).

The second attenuator at HED is installed just upstream of the CRL3 optics in the HED optics hutch. Here the filters are mounted on four motorized arms which hold six attenuators each. These 24 filters are either Si or CVD diamond with varying thicknesses $(0.025 \mathrm{~mm}-6.4 \mathrm{~mm}$ for $\mathrm{Si}$ and $0.1 \mathrm{~mm}-$ $1.6 \mathrm{~mm}$ for CVD diamond). A wide range of transmissions can be achieved by the insertion of filters in up to four arms simultaneously.

The pulse picker unit (PPU) can pick pulse trains at a maximum repetition rate of $10 \mathrm{~Hz}$. It is positioned just downstream of the HED XGM and consists of a rotating chopper disk made from a sandwich of $2 \mathrm{~mm} \mathrm{~B}_{4} \mathrm{C}$ and $3 \mathrm{~mm}$ Densimet with several openings across its circumference. This disk is rotated by a fast DC motor.

\section{Drivers}

\subsection{Intense X-ray pulses}

Assuming a typical performance of the SASE2 undulator at 6-10 keV of $2 \mathrm{~mJ}$ pulse energy (reduced to $\sim 1 \mathrm{~mJ}$ at the TCC due to the overall beamline transmission) in a duration of $25 \mathrm{fs}$ allows to create power densities, or peak intensities, in excess of $10^{17} \mathrm{~W} \mathrm{~cm}^{-2}$ when focused to $5 \mu \mathrm{m}$ FWHM. These intensities volumetrically excite solid-density matter at timescales shorter than a phonon period, and predominantly couple to certain atomic orbitals when the photon energy is chosen with
Table 3

Transmission, diffraction limit and intensity for the nanofocus CRLs.

\begin{tabular}{llll}
\hline & $\begin{array}{l}\text { Transmission } \\
(\%)\end{array}$ & $\begin{array}{l}\text { Diffraction } \\
\text { limit }(\mathrm{nm})\end{array}$ & $\begin{array}{l}\text { Intensity } \\
\left(10^{18} \mathrm{~W} \mathrm{~cm}^{-2}\right)\end{array}$ \\
\hline 20 CRLs & 50 & 122 & 2.1 \\
30 CRLs & 37 & 89 & 3.0 \\
40 CRLs & 28 & 73 & 3.3 \\
50 CRLs & 22 & 64 & 3.4 \\
\hline
\end{tabular}

respect to resonances or absorption edges (Vinko et al., 2012; Sperling et al., 2015; Yoneda et al., 2015).

Using the nanofocus setup, the diffraction-limited spot sizes and resulting intensities for a stack of Be CRLs with radii of curvature of $50 \mu \mathrm{m}$ and $300 \mu \mathrm{m}$ geometric aperture, a monochromatic (seeded) beam at $9 \mathrm{keV}, 500 \mu \mathrm{J}$ pulse energy (accounting for the upstream beamline transmission) are as given in Table 3.

While the achievable intensities are ten times higher than using CRL3, the excited volume is small and comparable with the mean free path of photo- and Auger electrons which will rapidly redistribute the deposited energy. The photon-energydependent transmission can be calculated by equation (48) of Lengeler et al. (1999) and depends on the absorption properties of the lens material and the related effective numerical aperture.

A unique possibility of European XFEL is X-ray excitation of a sample with a $\mathrm{MHz} \mathrm{X}$-ray burst. Free-standing or statically compressed samples (e.g. in a DAC) can be sequentially and volumetrically heated and simultaneously probed by X-ray techniques ( $c f$. Section 7).

While the delay between two subsequent pulses from the accelerator is limited to $222 \mathrm{~ns}$, in the near future the HED instrument will also provide two-pulse techniques with separations $<1 \mathrm{ps}$, either by two-color SASE with an electron chicane or by using an X-ray split-and-delay line (Mitzner et al., 2008; Wöstmann et al., 2013; Roling et al., 2017; Kärcher et al., 2021).

\subsection{Diamond anvil cells}

The provision of X-ray photon energies $>10 \mathrm{keV}$ enables the HED instrument to perform experiments in DACs. The DAC setup in the IC1 chamber is suited for emission spectroscopy-type experiments (Section 6.2.6), whereas the one in IC2 provides a dedicated platform for $\mathrm{MHz}$ X-ray diffraction in dynamic DAC compression and heating experiments (Sections 6.4 and 6.4.1) which are described in detail by Liermann et al. (2021). High temperatures (typically in the range 1000-10000 K) are generated either via double-sided pulsed laser heating or by X-ray heating, the latter by varying the repetition rate of the $\mathrm{X}$-ray pulses within a train. Dynamic compression is achieved in a piezo-driven dynamic DAC (dDAC) capable of compressing samples to megabar pressures on a millisecond time scale (Jenei et al., 2019), enabling the study of material behavior under intermediate strain rates between those achieved in static and shock compression experiments. In dynamic, laser-driven shock compression 
Table 4

Basic specifications of the pump-probe laser.

\begin{tabular}{lcll}
\hline Name & Central wavelength $(\mathrm{nm})$ & Repetition rate & Pulse duration (FWHM) \\
\hline Mode 1 (NOPA) & \multirow{2}{*}{800} & $100 \mathrm{kHz} @ 2 \mathrm{~mJ}$ & $15-300$ fs nearly transform limited \\
Mode 2 (amplifier for NOPA) & \multirow{2}{*}{1030} & $4.5 \mathrm{MHz} @ 0.05 \mathrm{~mJ}$ & $0.9 \mathrm{ps}$ (compressed), 500 ps (chirped) \\
& & $100 \mathrm{kHz} @ 35 \mathrm{~mJ}$, & $4.5 \mathrm{MHz} @ 1 \mathrm{~mJ}$
\end{tabular}

experiments, DACs can also be used to precompress materials in order to reach higher pressures at moderate temperatures which are not accessible by shock-compressing materials from ambient pressures (Brygoo et al., 2015; Loubeyre et al., 2012).

\subsection{Optical lasers}

5.3.1. ReLaX laser. The High-intensity Relativistic Laser at XFEL (ReLaX) is based on a commercial titanium sapphire laser system manufactured by Amplitude Technologies in France. The laser architecture follows a double chirped pulse amplification scheme, with temporal contrast enhancement using polarization filtering and spectral broadening by crosspolarized wave generation (XPW). It can deliver up to $300 \mathrm{TW}$ at $5 \mathrm{~Hz}$ repetition rate and $100 \mathrm{TW}$ at $10 \mathrm{~Hz}$ in nominal operation mode with pulses as short as 25 fs FWHM. The wavelength is $(800 \pm 40) \mathrm{nm}$. The whole laser chain has been designed with high redundancy of pump lasers and low fluence due to oversized optics in the compressed beam transport. The optical compressor, beam transport, and diagnostic package have been designed and manufactured through HIBEF by HZDR. Two loops on the wavefront using a deformable mirror are employed, one before and one behind the optical compressor. The commissioning and integration phase into the HED instrument was successfully completed in 2019. In addition to the main beam of ReLaX, low-energy probe beams are available, either for optical probing or timing cross-correlation with X-rays. The unfocused main beam diameter is $15 \mathrm{~cm}$. Experiments with the ReLaX laser are only possible in IC1 (see Section 6.2), which is equipped with an $f / \#=2$ off-axis parabola as final focusing optics, allowing multiple sample irradiation geometries, e.g. co-linear, $45^{\circ}$ or normal to the X-ray beam propagation direction. Sending the uncompressed pulse to IC1 was not foreseen and is currently technically impossible.

5.3.2. DiPOLE 100-X laser. The High Energy laser DiPOLE 100-X (Diode Pumped Optical Laser for Experiments) is an all diode-pumped $100 \mathrm{~J}$ class ytterbium:YAG based laser, manufactured by STFC CLF (Central Laser Facility) in the UK and the University of Oxford as part of the UK's contribution to the HIBEF UC (Phillips et al., 2019). The laser system delivers $100 \mathrm{~J}$ for a $10 \mathrm{~ns}$ pulse duration and $37 \mathrm{~J}$ for a $2 \mathrm{~ns}$ pulse duration at the fundamental wavelength $(1030 \mathrm{~nm})$. In addition it is capable of $10 \mathrm{~Hz}$ operation resulting in $\mathrm{kW}$ output of optical light. Since the main scientific use of DiPOLE 100-X is laser-driven shock and ramp compression, the laser provides pulse shaping capabilities with a resolution of $125 \mathrm{ps}$, allowing arbitrary waveforms ranging in duration from 2 to $15 \mathrm{~ns}$. Frequency doubling of the $1030 \mathrm{~nm}$ laser to
$515 \mathrm{~nm}$ with $60 \mathrm{~J}$ at $10 \mathrm{~Hz}$ for a $10 \mathrm{~ns}$ pulse has been demonstrated using a $60 \mathrm{~mm}$ large aperture lithium-triborate (LBO) crystal (Phillips et al., 2021); conversion efficiencies for a 2 ns pulse are expected to be similar. The laser is synchronized with the X-ray beam using the XFEL timing system which has an RMS jitter of approximately $10 \mathrm{ps}$. The temporal total temporal jitter of the laser beam against the X-ray beam including all components is not yet characterized, but expected to be better than $50 \mathrm{ps}$. We also plan an online monitoring of the arrival time of both laser and X-rays. Phase plates providing flat-top focal spot profiles ranging from 100 to $500 \mu \mathrm{m}$ diameter shall be provided. The DIPOLE 100-X laser will be available in both IC1 and IC2 with irradiation ranging from close to co-propagation $\left(22.5^{\circ}\right)$ to perpendicular.

The full capabilities of DiPOLE 100-X can be exploited in combination with the HIBEF-provided VISAR (see Section 6.7.1) system and the high-precision XRD platform at IC2 (see Section 6.4). The DiPOLE 100-X laser was delivered to European XFEL in 2019 and is currently being commissioned. The first user experiment is scheduled for 2022. Fig. 11 shows both the ReLaX and DiPOLE 100-X lasers in the laser bay.

5.3.3. Pump-probe laser. In addition to the HIBEF optical lasers, the pump-probe (PP) laser, developed at European XFEL (Palmer et al., 2019), offers intense laser pulses tailored to the unique time structure of the XFEL bunch pattern. This laser, which operates with the non-collinear optical parametric amplifier (NOPA) scheme, has a central wavelength of $800 \mathrm{~nm}$ with a close to Fourier-limited bandwidth for pulses down to 15 fs duration. The laser can deliver synchronized pulses in $10 \mathrm{~Hz}$ bursts of up to $600 \mu \mathrm{s}$ length and an intra-burst repetition rate up to $4.5 \mathrm{MHz}$. A maximum pulse energy of $\sim 2 \mathrm{~mJ}$ is available at a reduced intra-burst repetition rate of $100 \mathrm{kHz}$ (Table 4). The pulse duration can be adjusted

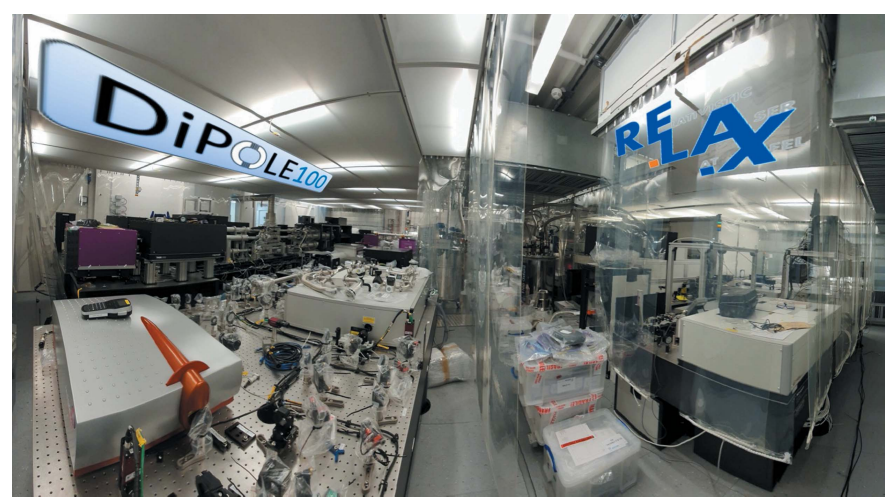

Figure 11

View into the HED laser bay with both HIBEF high-power lasers DIPOLE 100-X (left) and ReLaX (right). 
between 15 and 300 fs FWHM via bandwidth management while remaining close to the Fourier limit (no chirp). The PP laser is operational since 2020. Currently, the laser is operated with a single pulse energy of around $1 \mathrm{~mJ}$ with $100 \mathrm{kHz}$ repetition rate and pulse duration of $17 \mathrm{fs}$ FWHM. The second harmonic generation (SHG, $\lambda=400 \mathrm{~nm}$ ) is also available. The conversion efficiency to SHG is only about $15 \%$ at $15 \mathrm{fs}$ operation due to its large spectral bandwidth. In addition, the pump laser for the NOPA can be delivered to experiments upon request. This laser has a fundamental wavelength of $1030 \mathrm{~nm}$ with $\sim 2 \mathrm{~nm}$ bandwidth which results in $\sim 1 \mathrm{ps}$ pulse duration, or $\sim 500 \mathrm{ps}$ in chirped mode. This operation mode is particularly interesting for applications which require higher single pulse energies of up to $35 \mathrm{~mJ}$.

5.3.4. Synchronization of optical lasers and XFEL pulses. The optical lasers ReLaX, DiPOLE, and PP require spatial and temporal synchronization with the X-ray pulses which places high demands on environmental stability and an extensive online diagnostics. The linear accelerator provides pulses according to its own timing, and the various laser sources need to be synchronized relative to it. When lasers are synchronized using the accelerator's radiofrequency only, a jitter of about $300 \mathrm{fs}$ is observed (Kirkwood et al., 2019). To improve on this, a Master-Laser-Oscillator (MLO) provides a more stable timing reference. A dispersion-compensated, actively stabilized optical fiber link synchronizes the individual oscillators of the ReLaX and PP laser on a sub-10 fs level. Nevertheless, the subsequent amplification process in both the FEL and the optical lasers and their long beam transport will lead to additional timing jitter and long-term temporal drift. Therefore, a pulse-resolved online photon arrival monitor (PAM) is implemented to record the relative arrival time between the optical and the X-ray pulses at the $10 \mathrm{~Hz}$ repetition rate. The PAM is permanently installed about $10 \mathrm{~m}$ upstream of the IC1 sample position in the optics hutch, before the X-ray attenuator and CRL3. This allows the jitter measurements to be quasi-independent from the X-ray focusing scheme and attenuation level which may be varied during experiments. The obtained data can be later used for

\section{a) Spatial encoding}

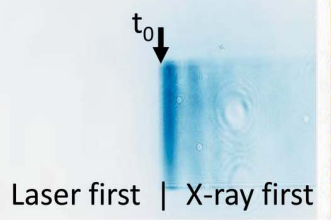

\section{b) Spectral encoding}

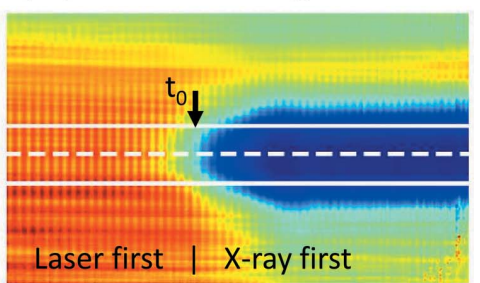

Figure 12

Typical images of PAM. (a) Spatial encoding: vertical versus horizontal position; time mapping results form an angle between the laser and the $\mathrm{X}$ ray on the sample $\left(2 \mu \mathrm{m}\right.$-thick $\left.\mathrm{Si}_{3} \mathrm{~N}_{4}\right)$. The temporal window (horizontal) is about $3.6 \mathrm{ps}$. (b) Spectral encoding image: lateral position versus the wavelength mapped to time by a chirped laser pulse collinear with the $\mathrm{X}$-ray perpendicular to the sample (100 $\mu \mathrm{m}$-thick YAG:Ce). The temporal window (horizontal axis of the image) is about $1.7 \mathrm{ps}$. Both show a shadow due to the X-ray-induced opacity change of the sample. At $t_{0}$ both beams are timed. The horizontal edges in $(a)$ result from the upstream partially closed power slits which are not influencing $(b)$. time-sorting and binning. The typical sample is $\mathrm{Si}_{3} \mathrm{~N}_{4}$ with $2 \mu \mathrm{m}$ thickness. This ensures a high $\mathrm{X}$-ray transmittance $(>90 \%$ at $>5 \mathrm{keV}$ and $>97 \%$ at $>8 \mathrm{keV}$ ) for experiments downstream. The PAM consists of two sample chambers which allows simultaneous measurements using spatial (Harmand $e t$ al., 2013; Riedel et al., 2013) and spectral encoding (Bionta et al., 2011) (Fig. 12) methods. While spatial encoding provides a better signal-to-noise ratio, the spectral encoding appears to be more robust against $\mathrm{X}$-ray pointing fluctuations. To ensure that PAM works at different $\mathrm{X}$-ray photon energies, $\mathrm{Si}_{3} \mathrm{~N}_{4}$ of 4 and $6 \mu \mathrm{m}$ thickness and a high- $Z$ material sample (e.g. YAG:Ce 10, $20 \mu \mathrm{m}$ thicknesses) are available, and are interchangeable during experiments.

The arrival timing jitter between the X-ray and the PP laser on PAM was measured to be 20-30 fs RMS.

\subsection{Pulsed magnet}

In 2021, the HIBEF UC will install a $750 \mathrm{~kJ}$ capacitor bank with a peak current of $100 \mathrm{kA}$ and pulsed magnets in different geometries: a horizontal bi-conical $60^{\circ}-20^{\circ}$ solenoid with peak fields of $60 \mathrm{~T}$, and a split-coil $(30 \mathrm{~T})$ for diffraction experiments exploiting the full equatorial plane. Both coil systems integrate an eddy current shield in order to minimize stray fields and resulting vibrations due to interactions with the environment. The coils are cooled by liquid-nitrogen bath cryostats and the sample cryostat provides temperatures between $4 \mathrm{~K}$ and $600 \mathrm{~K}$. The current design of the pulsed magnet setup represents a separate experimental platform optimized for peak field strengths and cryogenic temperatures. Thus, it cannot be used in conjunction with other drivers such as DACs or optical lasers. Furthermore, development of a phase retarder to control the polarization of the incident $\mathrm{X}$-ray beam and of the polarization analyser for the scattered $\mathrm{X}$-rays is currently ongoing.

\section{Experimental platforms \\ 6.1. Experimental hutch layout}

The experiment hutch enclosure is constructed from up to $100 \mathrm{~cm}$-thick heavy concrete walls, to anticipate the radiation and particles generated from the intense optical laser-matter interaction (e.g. using the ReLaX laser) and has been designed based on FLUKA simulations (Nakatsutsumi \& Tschentscher, 2013; Battistoni et al., 2015). Transport of large equipment in and out of the hutch is possible through a $3 \mathrm{~m}$-wide by $2.5 \mathrm{~m}$ high sliding door.

The floor plan of the $4 \mathrm{~m}$-high HED experiment hutch is shown in Fig. 13, and the X-ray beam path is $1400 \mathrm{~mm}$ above the hutch floor. The hutch is organized in two interaction areas (IAs): the X-rays enter IA1 by passing through a differential pumping section into the fixed interaction chamber (IC) 1 (see Section 6.2) and then proceed to IA2. All drivers except the pulsed magnet can be brought to the IC1 chamber. Furthermore, the ReLaX (Section 5.3.1) and DiPOLE (Section 5.3.2) laser beams are brought into the experiment hutch from the laboratory on the top floor through chicanes in the roof. On 


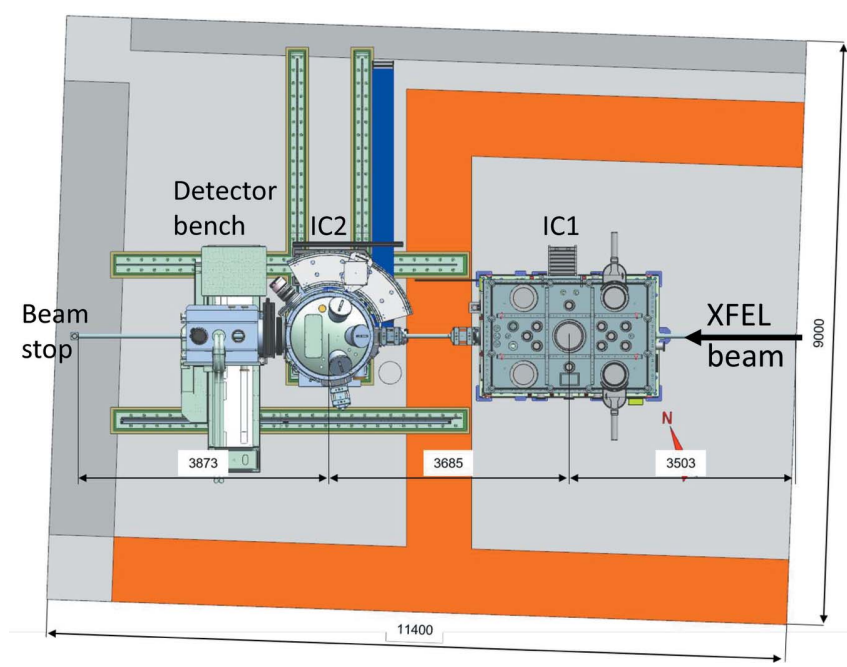

Figure 13

Floor plan of HED experiment hutch with the positions of IC1 (right, rectangular), IC2 (center, round) and the AGIPD on the detector bench on the left. The XFEL beam enters from the right. The dark-gray areas indicate hutch infrastructure, while the orange areas mark access paths. Dimensions are given in millimetres.

either side of the IC1 chamber are optical tables for optical laser diagnostics and optical sample diagnostics such as VISAR (Section 6.7.1).

The IA2 allows for various dedicated setups. Here, the IC2 chamber (Section 6.4) is permanently mounted on a rail system, which is embedded in the hutch floor, and can be brought into the beam, or parked by the wall. In the near future, IA2 will also host a goniometer for pulsed magnetic field experiments.

Downstream of IC1, rails run parallel to the X-ray beam path, carrying a $3 \mathrm{~m}$-wide detector bench. It can be located at any position between the rear end of IC1 and the beam stop at the hutch wall. The bench is optimized to minimize vibrations and equipped with spectrometers and detectors including VAREX or AGIPD (see Sections 6.4.4 and 6.4.3).

The standards for vacuum in the experiment chambers require clean conditions at $<10^{-4}$ mbar pressure (Schmidt \& Dommach, 2015). This volume is decoupled from the ultrahigh vacuum in the optics hutch and tunnels $\left(\sim 10^{-9}\right.$ mbar $)$ by a differential pumping stage (DPS) which allows window-less $\mathrm{X}$-ray operation. However, if the experiment requires ambient pressure, gate valves before IC1 and IC2 can be used to separate the chambers which are equipped with a $10 \mathrm{~mm}$ diameter, $100 \mu \mathrm{m}$-thick diamond window with excellent X-ray transmission.

\subsection{Interaction chamber 1}

IC1 is a large multi-purpose chamber, manufactured by TOYAMA. All laser drivers, a manifold of sample geometries, spectrometers and other diagnostics can be arranged in vacuum around the X-ray beam at the TCC. The chamber body is made from an $\mathrm{Al}$ alloy to avoid long-duration nuclear activation during laser-plasma experiments which could interrupt user operation. Perpendicular to the X-ray path, large hinged doors allow easy access. The primary access doors open into a clean tent providing a flow of filtered air.

As the X-rays at European XFEL are horizontally polarized, scattering is preferably measured in the vertical plane where the scattered signal is not reduced by polarization effects. Therefore IC1 provides a vertical breadboard with motorized rails in the shape of circular arcs with the TCC in their centers (see Fig. 14). The arc radii are $R=306,517$ and $750 \mathrm{~mm}$. Onto these, motorized carriages are mounted which can hold detectors and spectrometers of several $\mathrm{kg}$ weight. A central target mount and a horizontal breadboard are mechanically decoupled from each other and the vacuum chamber itself, and rest on separate granite supports. The dimensions of the horizontal breadboard are $2.3 \mathrm{~m} \times 1.4 \mathrm{~m}$, and the X-ray beam is $(349 \pm 1) \mathrm{mm}$ above its surface. It

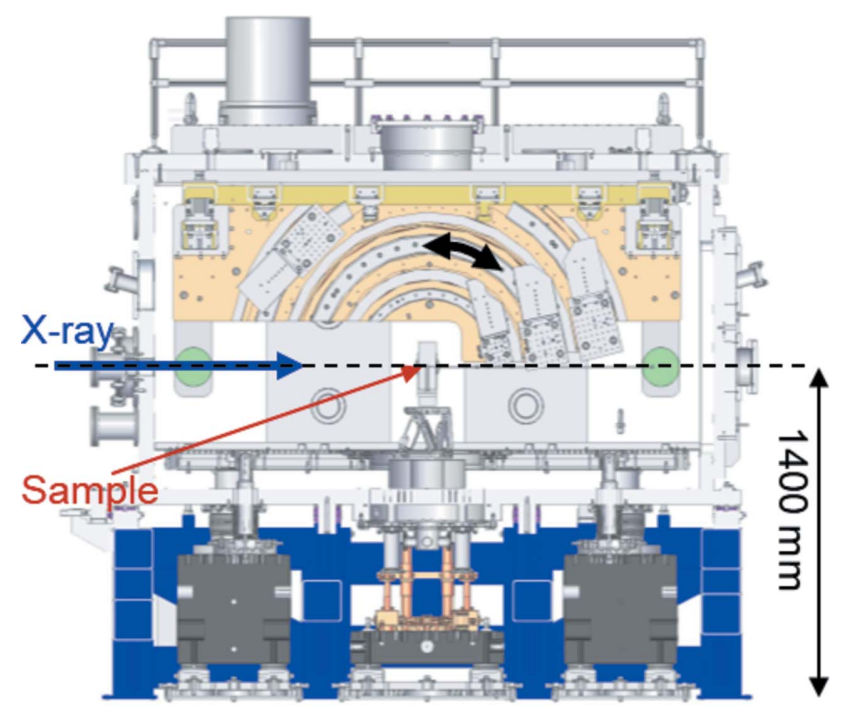

(a)

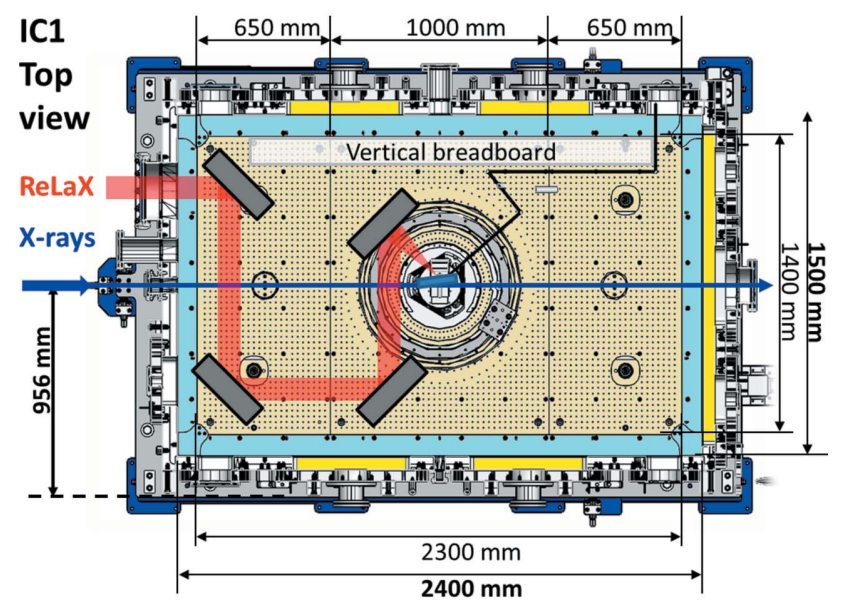

(b)

Figure 14

(a) Cut through the schematic of the IC1 target chamber showing the vertical breadboard with circular rails. (b) Top view of the interior of IC1; the access doors are at the bottom. In red, a typical beam path for the ReLaX laser is shown, and the back line indicates a sample viewing system. The beam transport of the DiPOLE-100X high-energy laser in IC1 is flexible and customized configurations are currently evaluated. 
provides M6 threaded holes in a $25 \mathrm{~mm}$ pattern. The IC1 walls are equipped with multiple feedthroughs with Japanese Industry Standards (JIS), ISO-K, and KF standards. On the roof of IC1, six turbomolecular pumps (HIPACE 800, Pfeiffer) with a pumping power of $790 \mathrm{l} \mathrm{s}^{-1}$ (for $\mathrm{N}_{2}$ ) pump the chamber through two large gate valves to $<10^{-5}$ mbar.

6.2.1. Sample tower. In the center of IC1, an electrically and mechanically insulated sample tower consists of (from top to bottom): horizontal and vertical linear stages (Fast Sample Scanner), Hexapod (H-824, Physik Instrumente), $360^{\circ}$ rotation stage (Goniometer 411, Huber Diffraktionstechnik), and a linear Y-axis (height) stage. It is possible to remove the Fast Sample Scanner to directly access the Hexapod. The scanner accommodates EUCALL (Appleby et al., 2017; see also Prencipe et al., 2017) standard sample holders and its travel ranges allows for $10 \mathrm{~cm} \times 10 \mathrm{~cm}$ effective sample area available (see Fig. 15). Within these limits, the sample mount can be freely customized to the shape, thickness, structure and orientation as required. A description and specification for each stage is summarized in Table 5.

6.2.2. Sample exchanger. Frequent sample replacement is required due to the high repetition rate of both the $\mathrm{X}$-ray beam and the optical laser systems. To mitigate the time for venting and pumping processes (20-30 minutes each), a robotic sample exchange system was constructed, consisting of a motorized sample exchange arm and a load-lock chamber on top of IC1, which is only compatible with EUCALL standard frames.

This system allows for the replacement of a frame mounted on the fast sample scanner, with one from the cassette in the load-lock, without breaking the vacuum. Each cassette can hold up to 16 sample frames with an overall thickness (including mounts/frames) $<10 \mathrm{~mm}$. For thicker samples, fewer frames may be stored accordingly. This system is currently under commissioning.

6.2.3. Microscopes. Questar. Two long-distance microscopes (QM 1 MKIII, Questar Cooperation) are mounted externally to IC1, upstream and downstream from the sample. The focus is motorized. Both telescopes are positioned at a working distance of $\sim 1.5 \mathrm{~m}$ from the sample and have a field of view of $\sim 13 \mathrm{~mm} \times 8 \mathrm{~mm}$. They have a spatial resolution of $20 \mu \mathrm{m}$, with a sampling of about $6.5 \mu \mathrm{m}$ per pixel. Motorized shutters at each microscope protect the optics and camera sensors from high intensity radiation generated during optical laser experiments.

In-Line Microscope (ILM). Two ILMs are mounted in IC1 on multi-axis translation stages. The upstream ILM consists of a $10 \times$ magnification microscope objective with $2 \mathrm{~mm}$ field of view, $33 \mathrm{~mm}$ working distance, and a $2.5 \mathrm{~mm}$ central hole which allows the incoming X-ray beam to pass through. The downstream ILM can accommodate standard objectives with various magnifications $($ e.g. $4 \times, 10 \times$ or $20 \times)$, to image the sample or characterize laser focal spots. The multi-axis stages can be moved on a horizontal circular rail around the sample, and can be adjusted to micrometere precision.

6.2.4. Cryogenic liquid jet targets. Liquid jets and cryogenic liquid jets have great potential to deliver replenishing
Table 5

Specifications of the sample tower.

\begin{tabular}{|c|c|c|c|}
\hline Name & Travel range & Speed & Repeatability \\
\hline $\begin{array}{l}\text { Fast Sample Scanner } \\
\text { horizontal }\end{array}$ & $62.5 \mathrm{~mm}$ & $0.1-20.0 \mathrm{~mm} \mathrm{~s}^{-1}$ & $2 \mu \mathrm{m}$ \\
\hline $\begin{array}{l}\text { Fast Sample Scanner } \\
\text { vertical }\end{array}$ & $57.5 \mathrm{~mm}$ & $0.1-15.0 \mathrm{~mm} \mathrm{~s}^{-1}$ & $2 \mu \mathrm{m}$ \\
\hline PI Hexapod horizontal & $\pm 22.5 \mathrm{~mm}$ & $1 \mathrm{~mm} \mathrm{~s}^{-1}$ & $0.1-0.5 \mu \mathrm{m}$ \\
\hline PI Hexapod vertical & $\pm 12.5 \mathrm{~mm}$ & $1 \mathrm{~mm} \mathrm{~s}^{-1}$ & $0.1-0.5 \mu \mathrm{m}$ \\
\hline $\begin{array}{l}\text { PI Hexapod rotation } \\
\text { (yaw, pitch) }\end{array}$ & $\pm 7.5^{\circ}$ & $11 \mathrm{mrad} \mathrm{s}^{-1}$ & $3 \mu \mathrm{rad}$ \\
\hline $\begin{array}{l}\text { PI Hexapod rotation } \\
\text { (roll) }\end{array}$ & $\pm 12.5^{\circ}$ & $11 \mathrm{mrad} \mathrm{s}^{-1}$ & $3 \mu \mathrm{rad}$ \\
\hline $360^{\circ}$ rotation stage & $360^{\circ}$ & N/A & $<10 \mu \mathrm{rad}$ \\
\hline Height adjustment stage & $150 \mathrm{~mm}$ & N/A & $<0.3 \mu \mathrm{m}$ \\
\hline
\end{tabular}

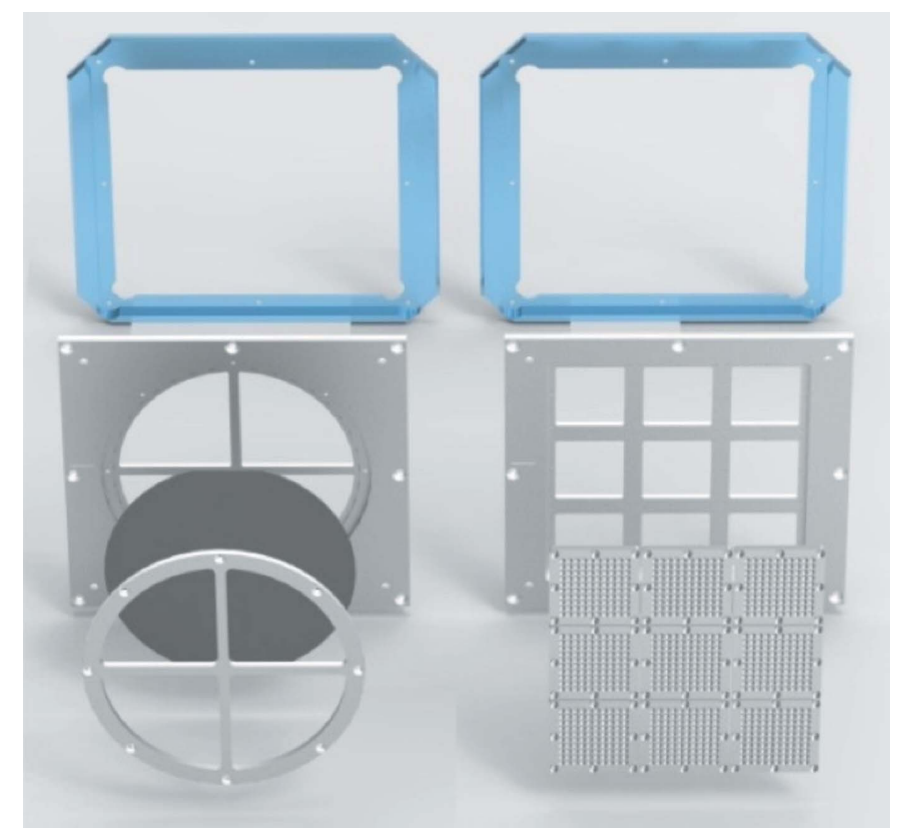

Figure 15

EUCALL standard frame design available at HED. The outer (blue) frame is unique to HED. The inner-frames (in gray/silver) can be customized for each experiment. This standard is employed at other beamlines at the EuXFEL, as well as other facilities such as ESRF and ELI beamlines.

targets for high-repetition-rate experiments without causing degeneration of laser optics by target debris (Kim et al., 2016). Moreover, they provide solid density matter which under ambient conditions only exists in the gas phase, and hence give experimental access to scientifically interesting samples such as hydrogen, methane, water and helium (Obst et al., 2017; Göde et al., 2017). The cryogenic jet platform implemented in IC1 will issue well characterized jets of cylindrical and sheet geometries with micrometre position accuracy and $<10 \mu \mathrm{m}$ diameters. Depending on the pumping speed and the gas properties, the maximum flow is limited to rates of about $500 \mathrm{sccm}\left(1 \mathrm{sccm}=0.016 \mathrm{mbar}^{1 \mathrm{~s}^{-1}}\right)$ which allow target crosssections of up to $100 \mu \mathrm{m} \times 100 \mu \mathrm{m}$ with $100 \mathrm{~m} \mathrm{~s}^{-1}$ flow velocities. Larger jets with lateral dimensions exceeding $10 \mu \mathrm{m}$ will be realized using a jet dump, currently under development. 
6.2.5. X-ray spectrometers. Spectroscopy applications studying extreme states require vacuum conditions and sufficient flexibility for excitation methods and setup of the various technique-dependent spectrometers. In IC1, X-ray Emission Spectroscopy (XES) and Inelastic X-ray Scattering (IXS), both for $\mathrm{meV}$ and $\mathrm{eV}$ resolution, are provided. For in-vacuum use in IC1, we have designed highly efficient spectrometers (Preston et al., 2020) using cylindrical mosaic crystals in von Hámos geometry (von Hámos, 1934). Highly Annealed Pyrolytic Graphite (HAPG) crystals (Zastrau et al., 2013) with thicknesses of $40 \mu \mathrm{m}$ or $100 \mu \mathrm{m}$ or Highly Oriented Pyrolitic Graphite (HOPG) (Zastrau et al., 2012) with thickness $100 \mu \mathrm{m}$ can be used, with radii of $50 \mathrm{~mm}$ or $80 \mathrm{~mm}$. The spectrometer is designed to use either an ePix100 or Jungfrau detector. The HAPG crystals have demonstrated (Preston et al., 2020) to reach resolving powers of $E / \Delta E \leq 2800$ at photon energies between 5 and $10 \mathrm{keV}$. An example spectrum of $\mathrm{Cr} K \alpha$ is shown in Fig. 16. Fig. 17 shows these spectrometers mounted in IC1, together with three diced analyzers (see below).

Furthermore, in conjunction with the aforementioned highresolution $\mathrm{Si}(533)$ monochromator (see Section 4.3), several diced analyzer crystals can be coupled with a detector to provide $40 \mathrm{meV}$ spectral resolution at $7495 \mathrm{eV}$. As shown in Fig. 18, this is sufficient to resolve inelastic X-ray scattering from phonons in solids, or ion-acoustic waves in plasmas (Wollenweber et al., 2021; Descamps et al., 2020).

6.2.6. Diamond anvil cell platform in IC1. The DAC setup in IC1 is built for $\mathrm{MHz}$ spectroscopy at high pressure and temperatures. While static high pressures are generated by the DAC itself, high temperatures in IC1 are achieved via X-ray heating, by varying the repetition rate or intensity of the X-ray pulses within a $\mathrm{MHz}$ pulse train. Future developments of this

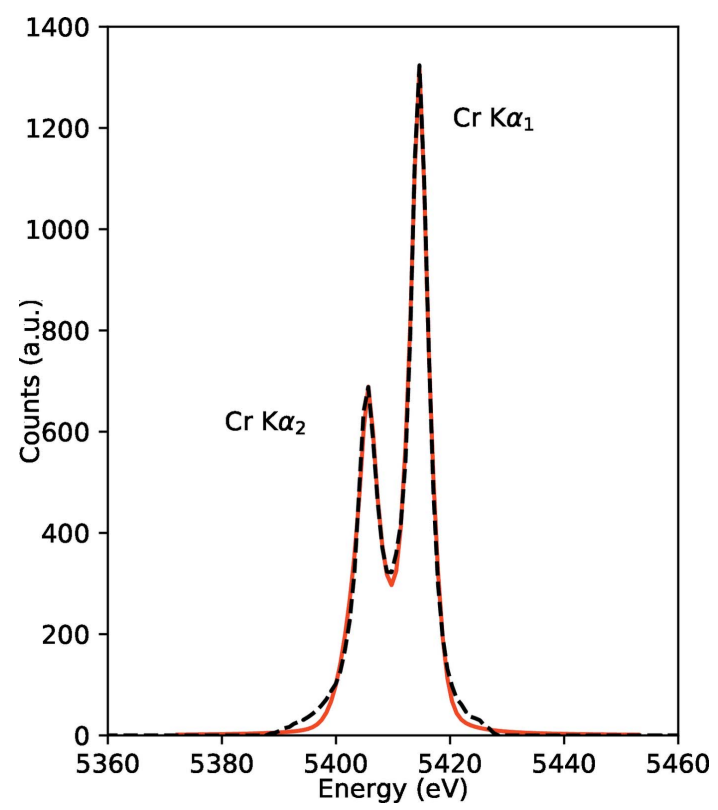

Figure 16

$K \alpha$ fluorescence of a $5 \mu \mathrm{m} \mathrm{Cr}$ foil irradiated by an $10 \mu \mathrm{m}$ X-ray FEL spot for calibration purposes (black-dashed) with fit (red). The spectral broadening of the lines by the $80 \mathrm{~mm}$ radius-of-curvature and $40 \mu \mathrm{m}$ thick HAPG crystal is $\sim 2 \mathrm{eV}$. Reproduced from Preston et al. (2020).

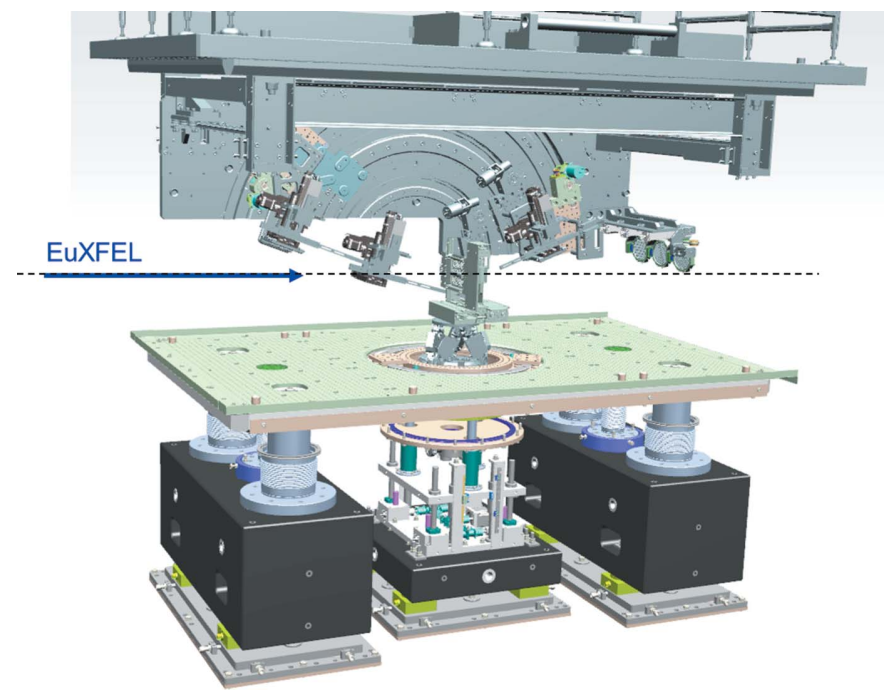

Figure 17

3D schematics of up- and downstream HAPG spectrometers and three diced analyzers mounted on the curved rail system in IC1, leaving the horizontal breadboard free for laser optics and further instrumentation.

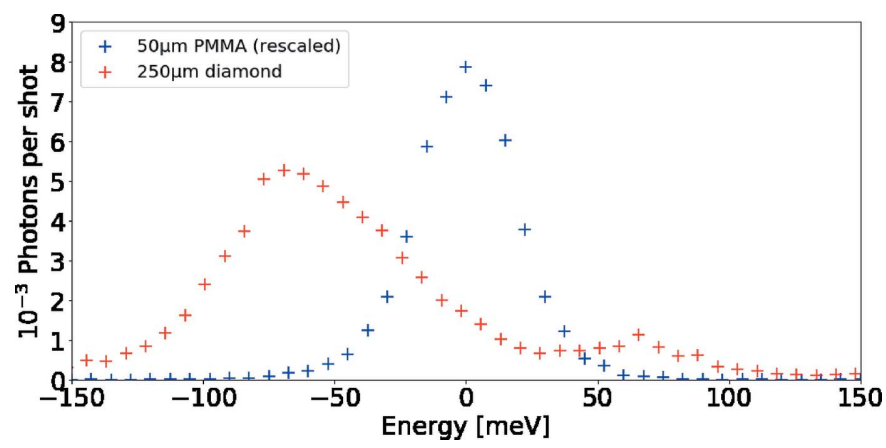

Figure 18

The spectra of the Si(533)-monochromated beam scattered from PMMA (elastic, blue) and single crystal diamond (inelastic, red) resolved with a diced Si (533) analyzer crystal. For better comparison, the elastic signal is reduced by a factor of ten. For diamond, the peaks correspond to phonon creation and annihilation. The spectrum is reproduced from Wollenweber et al. (2021).

experimental platform may include temperature measurements by optical diagnostics such as the portable system with streaked optical pyrometry (Section 6.7.2) and/or multi-color pyrometry (von Hámos, 1934). The X-ray spectrometer has been designed, built and contributed by the University of Dortmund, and is tailored for emission from matter contained in a diamond anvil cell. It comprises four vertically stacked analyzer crystals of either $\mathrm{Si}(111)$ or $\mathrm{Si}(531)$ with a radius of curvature of $250 \mathrm{~mm}$ and a size of $110 \mathrm{~mm} \times 20 \mathrm{~mm}$, covering an energy range of $6-8 \mathrm{keV}$ with a resolution of $0.3-0.4 \mathrm{eV}$ (Klementiev \& Chernikov, 2020).

\subsection{Small X-ray detectors}

The HED instrument is offering compact and vacuum compatible X-ray detectors. All detectors are suitable for X-ray diffraction, and can be used in conjunction with the aforementioned spectrometers. They can be positioned inside 
Table 6

Detector specifications available at IC1.

\begin{tabular}{llllll}
\hline $\begin{array}{l}\text { Detector } \\
\text { name }\end{array}$ & $\begin{array}{l}\text { Pixel size } \\
(\mu \mathrm{m})\end{array}$ & $\begin{array}{l}\text { Noise } \\
(\mathrm{adu})\end{array}$ & $\begin{array}{l}\text { Gain } \\
\left(\mathrm{eV} \mathrm{adu}^{-1}\right)\end{array}$ & Dynamic range & $\begin{array}{l}\text { Sensor size } \\
(\text { pixels })\end{array}$ \\
\hline ePix100 & $50 \times 50$ & $<4$ & 54 & 100 photons @ $8 \mathrm{keV}$ & $704 \times 768$ \\
ePix100H & $25 \times 100$ & $<4$ & 54 & 100 photons @ $8 \mathrm{keV}$ & $176 \times 1536$ \\
Jungfrau & $75 \times 75$ & $<15$ & 20 & $10^{4}$ photons @ $12 \mathrm{keV}$ & $512 \times 1024$ \\
\hline
\end{tabular}

platforms: one for DAC experiments [Fig. 20(b)], and one for dynamic laser compression experiments using the DiPOLE 100-X laser (Fig. 21).

The IC2 chamber has an outer diameter of $1360 \mathrm{~mm}$ and a height of $1520 \mathrm{~mm}$. Further details are given by Liermann et al. (2021). The chamber is located in IA2 and can be moved

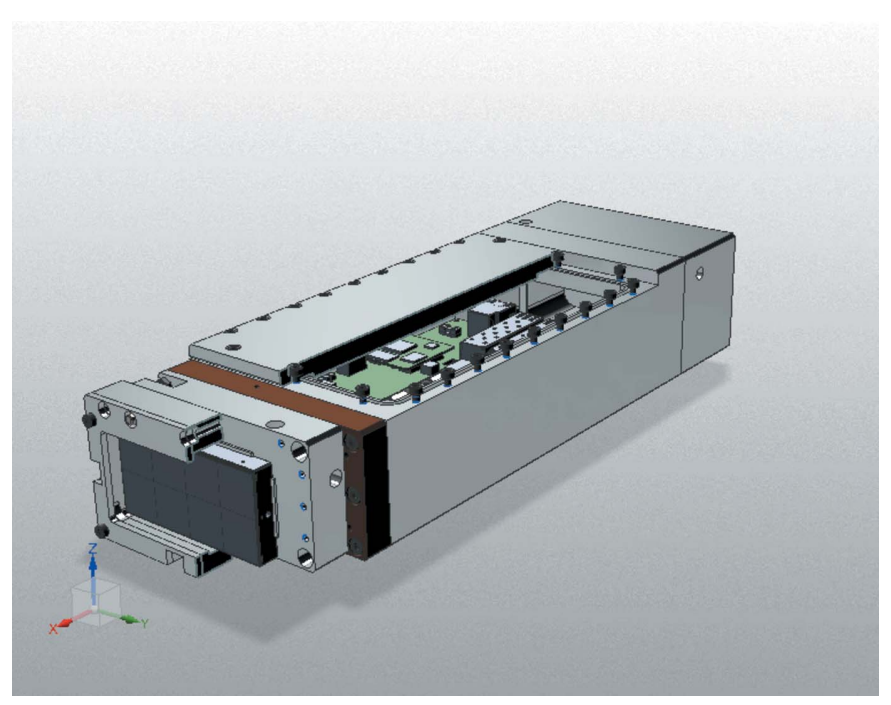

Figure 19

Model of the Jungfrau detector in an air-box, developed at the HED Instrument for in-vacuum operation.

IC1 using the vertical breadboard and motorized translation systems. In air, the detector bench in IA2 can be used to achieve larger sample-detector distances of up to $7 \mathrm{~m}$ for small-angle X-ray scatting (SAXS), radiography, or phase contrast imaging (PCI).

The detectors inside IC1 are the ePix100 (Blaj et al., 2016; Klačková et al., 2019), ePix100H hammerhead (Blaj et al., 2019), and the Jungfrau (Mozzanica et al., 2018). Their specifications are summarized in Table 6. The HED instrument currently offers two vacuum-compatible ePix100 modules to users, while two more modules with hammerhead sensors are foreseen. A dedicated air-box housing developed at European XFEL (Fig. 19) enables also the Jungfrau detector to be operated in vacuum. The Jungfrau features automatic gain switching of each pixel which allows detection of single photon events and high signal levels of up to $1000012 \mathrm{keV}$ photons in a single image (Redford et al., 2018, 2020). In total, four single Jungfrau modules are available and two modules can be combined into a larger detector with sensor size $2048 \times 516$ or $1024 \times 1024$ pixels with an inter-modular gap of $4.5 \mathrm{~mm}$ and $2.5 \mathrm{~mm}$, respectively.

\subsection{Interaction chamber 2}

IC2 [Fig. 20(a)] is optimized for diffraction experiments, exploiting the high photon energies and $\mathrm{MHz}$ time structure of the X-rays for the study of materials under extreme pressures, temperatures and strain rates. It hosts two experimental between the operating and parking position on a rail system. The vacuum system allows for turnaround times below 30 minutes including venting, pumping and sample exchanges. IC2 can be coupled with either of two detector systems: the AGIPD 1M detector (Section 6.4.3), designed to resolve individual pulses at the minimum bunch spacing of the X-ray at $222 \mathrm{~ns}$, and a twin configuration of two Varex flat-panel detectors (Section 6.4.4) for maximum gapless coverage at $10 \mathrm{~Hz}$ repetition rate in an EMP- and debris-resistant housing.

6.4.1. Diamond anvil cell platform in IC2. This DAC platform [Fig. 20(b)] is designed to explore $\mathrm{MHz}$ diffraction for $\mathrm{X}$-ray heating, pulsed laser heating, and dynamic compression experiments using piezoelectric drivers. A sample stack provides all motorizations that are required to align a DAC in the center of rotation, relative to the detector and X-ray beam. Stability and resolution were optimized for studies of micrometre-sized samples with beam sizes down to $100 \mathrm{~nm}$. Up to six conventional or three dynamic DACs can be mounted on carousel type sample exchangers. The DAC platform can be combined with a double-sided laser-heating setup which integrates sample observation, near infrared pulsed laser heating, and streaked optical pyrometry in a coaxial design. The addition of a four-channel multi-color spectrometer for fast, high-sensitivity pyrometry is planned.

6.4.2. Dynamic laser compression platform. The dynamic laser compression platform was conceived for maximum flexibility in the geometry between the DiPOLE laser, VISAR diagnostics (Section 6.7.1), and X-ray diffraction (Fig. 21). The sample stack was build around the EUCALL sample frame system. In addition, it features mounting interfaces for user-

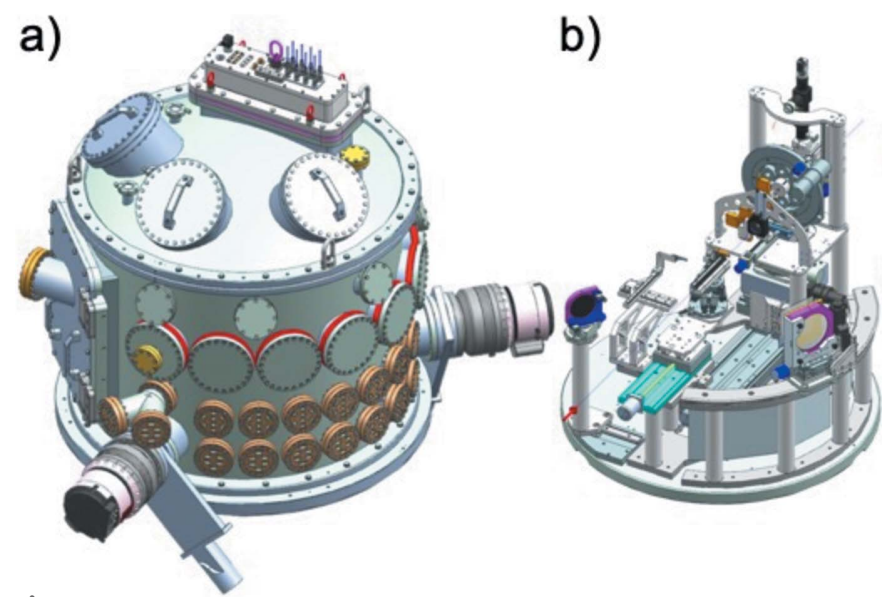

Figure 20

Interaction chamber IC2. (a) Vacuum chamber. (b) Platform for highpressure research with diamond anvil cells. 

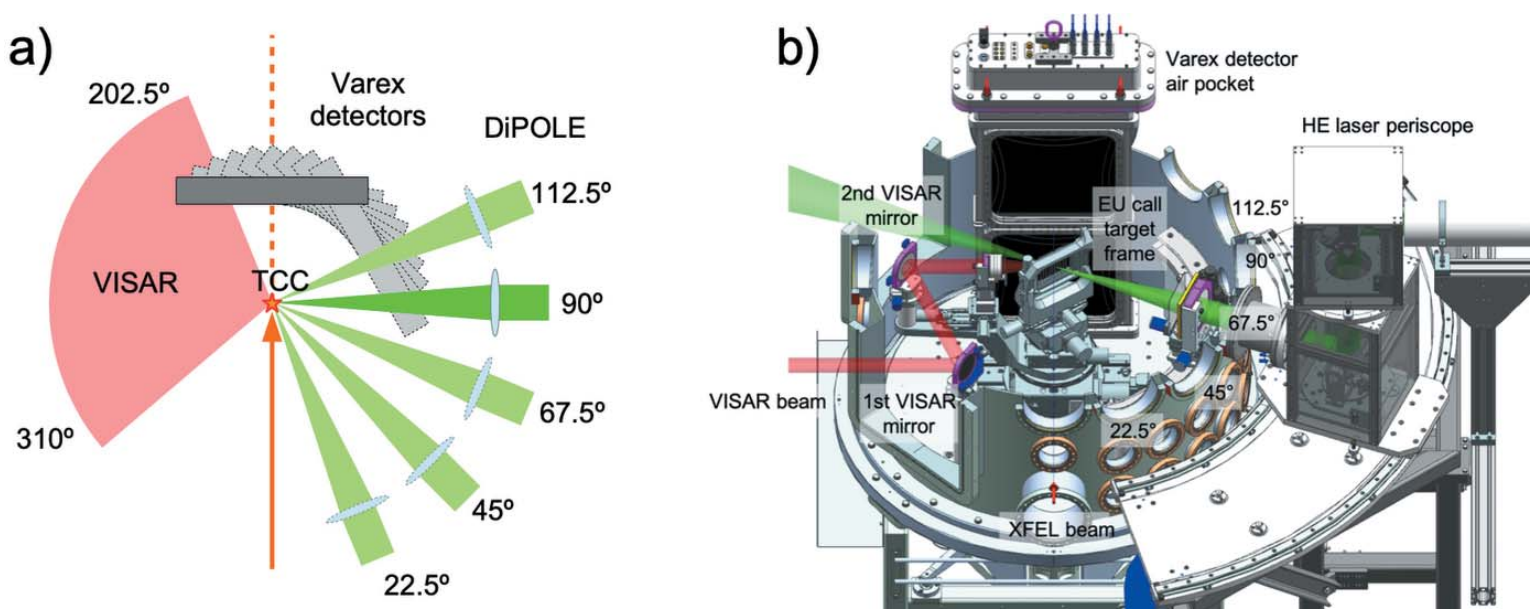

Figure 21

Dynamic laser compression platform optimized for flexible experimental geometries. For the drive laser, this is realized through five individual laser entrance ports at angles of $22.5^{\circ}, 45^{\circ}, 67.5^{\circ}, 90^{\circ}$, and $112.5^{\circ}$ with respect to the incident X-ray beam, to which the DiPOLE beam transport is coupled via an external periscope system. The VISAR beam enters the chamber through a single port and covers an angular range from $202.5^{\circ}$ to $310^{\circ}$ via a pair of fixed and mobile mirrors inside the chamber. The Varex detector system can be rotated around the interaction point in steps of $7.5^{\circ}$.

supplied sample delivery systems such as tape targets, or static precompression devices. For sample observation, Questar long-focal-distance microscopes aimed at the TCC can be installed on five ports at $0^{\circ}, 34^{\circ}, 79^{\circ}, 124^{\circ}, 280^{\circ}$ in the horizontal, with an observation angle of $18^{\circ}$ with respect to the equatorial plane.

6.4.3. AGIPD detector. A dedicated 1 megapixel version of the Adaptive Gain Integrating Pixel Detector (AGIPD) (Allahgholi et al., 2019) will be integrated in order to perform diffraction experiments at the X-ray pulse repetition rate of 4.5 $\mathrm{MHz}$ within a pulse train [Fig. 22(a)]. The AGIPD features automatic gain switching covering a dynamic range from single photon sensitivity (at $12 \mathrm{keV}$ ) up to $10^{4}$ photons per pixel. Up to 352 frames per train (with adjustable bunch pattern) are acquired in analog pixel-based storage and read out at the train repetition rate of $10 \mathrm{~Hz}$. While the initial version will be delivered with a $500 \mu \mathrm{m} \mathrm{Si} \mathrm{sensor,} \mathrm{DESY} \mathrm{FS-DS} \mathrm{is} \mathrm{currently}$ developing an electron collecting high- $Z$ version for increased efficiency at high $\mathrm{X}$-ray energies. The 1 megapixel version consists of sixteen $128 \times 512$-pixel modules in an $8 \times 2$ arrangement. The system is integrated in an independent vacuum chamber, which is connected to IC2 through a DN 500 gate valve and bellow system, in order to keep the detector cooled and biased when the interaction chamber has to be vented for sample exchanges. Inside its chamber, the detector is mounted on a positioning unit, in order to extend the sensor area into the interaction chamber for maximum coverage of up to $2 \theta=40^{\circ}$ at sample-detector distance (SDD) $=150 \mathrm{~mm}$ corresponding to $Q=9 \AA^{-1}$.

6.4.4. Varex detector. The Varex twin-detector system is designed to provide maximum gapless coverage and high quantum efficiency at high X-ray energies for DAC and shock experiments in IC2 at the bunch-train repetition rate of $10 \mathrm{~Hz}$ [Fig. 22(b)]. These specifications are matched by two Varex XRD 4343 CT flat-panel detectors with scintillator panels consisting of CsI:Tl oriented needle crystals which are bonded to a $2880 \times 2880$ pixel $(140 \mu \mathrm{m} \times 140 \mu \mathrm{m})$ TFT-diode array with an active surface of $432 \mathrm{~mm} \times 432 \mathrm{~mm}$. In order to reach $2 \theta$ angles of $64.5^{\circ}$ in the vertical, the detectors need to be placed inside IC2 at a sample-detector distance of $220 \mathrm{~mm}$. This is achieved by insertion of two of these detectors into an air pocket equipped with thin $(400 \mu \mathrm{m}) \mathrm{Al}$ windows through a dedicated lid for IC2. A twin configuration with a horizontal gap in the equatorial plane was chosen in order to avoid parasitic scattering from a beamstop and provide direct access to the transmitted beam for additional downstream diagnostics (e.g. intensity monitors, SAXS, and PCI).

\subsection{Goniometer}

A five-circle goniometer optimized for diffraction experiments in horizontal geometry with a load capacity of $300 \mathrm{~kg}$ is foreseen. It carries the magnet/sample cryostat assembly and can be reproducibly placed at IA 2 by the use of kinematic mounts, alternatively to the interaction chamber 2 . On the detector arm three different detector systems are planned: (1) an AGIPD module taking advantage of the pulse structure of the EuXFEL up to $4.5 \mathrm{MHz}$, (2) small pixel area detector module collecting diffraction data with higher angular resolution, and (3) analyser crystal for polarization-dependent scattering experiments.

\subsection{Equipment operation under harsh conditions}

Both the JUNGFRAU detectors in IC1 and the VAREX detector in IC2 are designed to operate together with the highintensity (IC1) or high-energy laser (IC1 and IC2). The interaction of these lasers creates electromagnetic pulses (EMP) and secondary radiation. The detectors are operated in a vacuum-tight metal housing which acts as a Faraday cage. The JUNGFRAU detectors in IC1 show stabile operation during laser-solid interaction at intensities $10^{20} \mathrm{~W} \mathrm{~cm}^{-2}$. The more sensitive ePIX100 and AGIPD detectors are currently not compatible with high-intensity and high-energy laser experiments. 


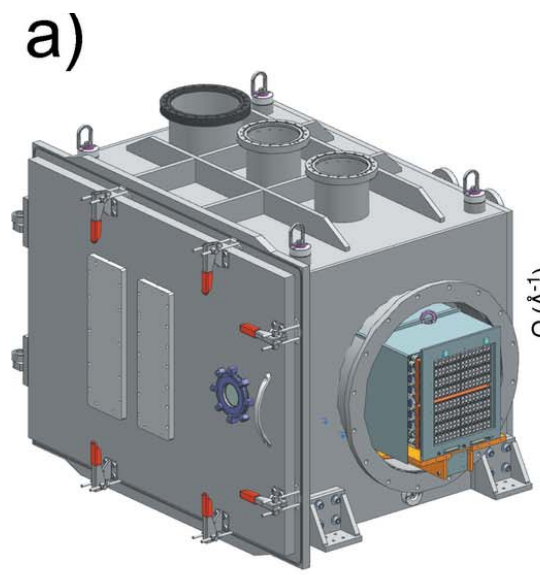

b)

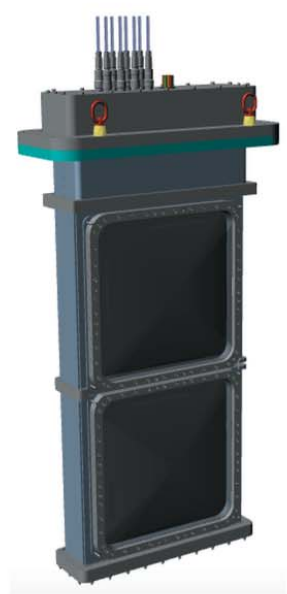

HIBEF AGIPD 1M SDD = $150 \mathrm{~mm}$

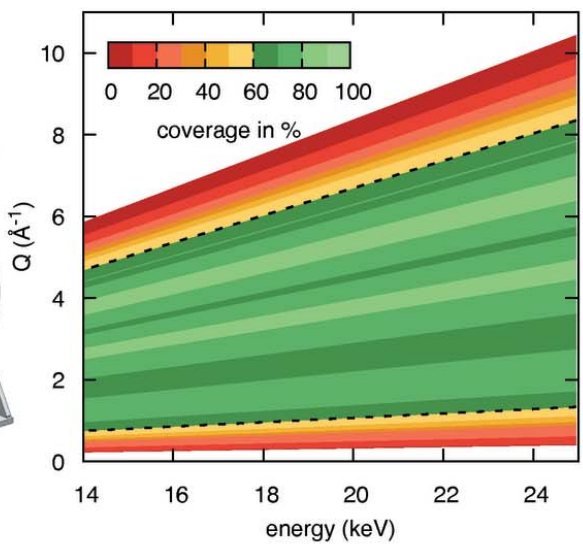

Varex twin configuration SDD $=220 \mathrm{~mm}$

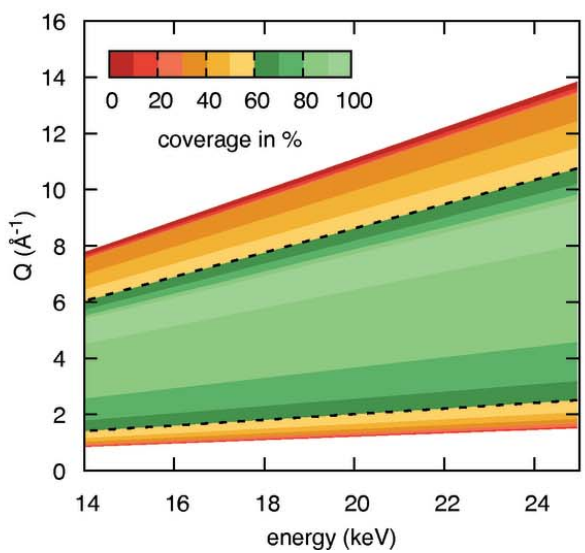

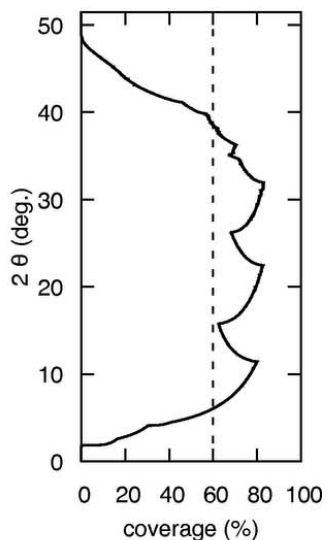

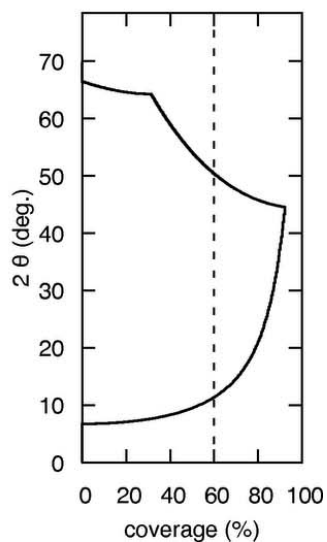

Figure 22

(a) HIBEF AGIPD 1M detector and coverage as a function of X-ray energy. (b) Varex twin detector system and coverage as a function of X-ray energy for the downstream position.

\subsection{Optical diagnostics}

In order to characterize the macroscopic state of dynamically compressed or excited matter, several optical diagnostics developed for the investigation of laser-matter interactions have been implemented. This is important for two reasons. First, the microscopic behavior of matter measured with $\mathrm{X}$-rays can be related to macroscopic properties. Second, these diagnostics can measure the macroscopic evolution of the material over a large temporal and spatial scale, thus relating the point in time measurement of the X-rays with, for example, the compression history of a sample.

6.7.1. Velocity Interferometer System for Any Reflector VISAR. In order to characterize laser-shocked samples, the HIBEF UC has contributed a VISAR system (Barker \& Hollenbach, 1965). VISAR enables the measurement of shock or interface velocities ranging from less than $100 \mathrm{~m} \mathrm{~s}^{-1}$ to above $50 \mathrm{~km} \mathrm{~s}^{-1}$ with a temporal resolution better than $10 \mathrm{ps}$. The VISAR system has in total three parallel arms to cover a wide range of detectable velocities which can range from $0.3 \mathrm{~km} \mathrm{~s}^{-1}$ fringe ${ }^{-1}$ to $40 \mathrm{~km} \mathrm{~s}^{-1}$ fringe ${ }^{-1}$. Two arms operate at $532 \mathrm{~nm}$ and one at $1064 \mathrm{~nm}$, permitting the simultaneous measurement of reflectivities at two different wavelengths.
The system is able to record temporal windows as long as $50 \mathrm{~ns}$ and can adapt the field of view from $250 \mu \mathrm{m}$ to $2 \mathrm{~mm}$ whilst keeping the full $F / 2.5$ numerical aperture of the optical system.

The VISAR is available in both IC1 and IC2; the beam path from IC2 to the VISAR system is routed through IC1. In IC1, similar optics as in IC2 will be used to pick up the reflection from the target surface and thus allow to use the same VISAR system for experiments in IC1.

6.7.2. Streaked Optical Pyrometry - SOP. Streaked Optical Pyrometry (SOP) is an instrument to deduce the temperature of a sample by analysing its emitted optical radiation. The temperature is either deduced from fitting the spectral intensity distribution to gray body radiation or the absolute radiation intensity at a given wavelength. The latter method has higher sensitivity and provides spatial information but requires a reflectance measurement, which can be provided by the VISAR system. Using a streak camera as a detector permits recording the temperature of the sample over time spans ranging from $1 \mathrm{~ns}$ to $100 \mathrm{~ms}$ with the possibility of sub-ns temporal resolution. The SOP system can be used in both interaction areas for various experiments such as pulsed laser heating, shock compression or isochoric heating. 
The SOP system specifically designed for IC2 detects thermal or fluorescence radiation emitted from the interaction of the XFEL pulses with a DAC sample, or during pulsed laser heating. The system features a HAMAMATSU streak camera with S-20 photocathode coupled to an optical spectrometer. It is placed outside the IC2, along with optics for high magnification microscopes for DAC sample observation, illumination, filtering and alignment. The spectral range for the optical spectrometer covers $440-850 \mathrm{~nm}$.

6.7.3. Fourier Domain Interferometry - FDI. Fourierdomain interferometry (FDI) (Geindre et al., 1994) enables the measurement of polarization-resolved reflectivity, phase shifts in the reflected probe due to plasma expansion or due to changes in refractive index via electronic excitation. The HED FDI system is available to users and consists of a 1:1 imaging spectrometer with an upstream Mach-Zehnder interferometer. It allows to measure the velocity of the critical density surface with $\sim 10 \mathrm{~nm}$ and a few 100 fs resolution in a $\sim 10$ ps temporal window.

\section{Methods and experiments}

The HED instrument supports a variety of ultrafast X-ray methods as summarized in Appendix A. XRD can be realized by positioning in-vacuum detectors in IC1 at the required geometry to cover dedicated scattering angular ranges. Designed for precision diffraction, IC2 provides larger detectors in forward-scattering, which in combination with hard X-rays allows radial patterns for particle distribution functions or texture analysis to be recorded.

In general, IC1 with its larger volume and two breadboards is optimized for spectroscopic applications. The HAPG spectrometers (Section 6.2.5) can be used for both XES and IXS. We also provide perfect crystals for a dedicated von Hámos analyzer for measuring $K$-shell emission from the $3 d$ transition metals $\mathrm{Cr}, \mathrm{Mn}$, $\mathrm{Fe}, \mathrm{Co}, \mathrm{Ni}, \mathrm{Cu}$ and $\mathrm{Zn}$. The spectrometer can be combined with a DAC sample environment and isochoric X-ray heating, which opens up the unique possibility to probe the electronic state at high pressures and temperatures of solid and liquid materials, e.g. that are of geological relevance.

$\mathrm{X}$-ray imaging is achieved by placing a nanometre X-ray focus upstream of the sample (by using the nanofocusing CRL4) and an on-axis projection onto a detector. The distance to the target needs to be sufficiently large for magnified PCI with high spatial resolution. The imaging performance depends also on several other parameters such as X-ray wavelength, focus and feature size, and sample properties (Schropp et al., 2015; Hagemann et al., 2021). Both
IC1 and IC2 qualify for PCI; however, IC1 allows for more propagation between sample and detector.

In order to illustrate the capabilities of the HED instrument, we highlight a few showcase experiments.

\subsection{X-ray heating in DACs}

Increasing the temperature in samples under pressure solely by exposing them to the $\mathrm{MHz} \mathrm{X}$-ray pulse train (Meza-Galvez et al., 2020) is an interesting alternative to the resistive or laser-heated DAC experiments (Spiekermann et al., 2020) because of the possibility to suppress chemical reactions and contamination of the sample from the diamond anvils. Highprecision diffraction experiments IC2 coupled with simultaneous SOP provided the temperature evolution and verified sample stability. The DAC assembly proved to be robust in the XFEL beam, which will in future enable novel pulse-resolved diffraction experiments at extreme conditions including high strain rates using $\mathrm{MHz}$ detectors.

Recently, Hwang et al. (2021) observed the ultrafast synthesis of $\varepsilon-\mathrm{Fe}_{3} \mathrm{~N}_{1+x}$ in a DAC from $\mathrm{Fe}$ and $\mathrm{N}_{2}$ under pressure using serial exposures of $17.8 \mathrm{keV}$ pulses, using the IC2 DAC platform. When the sample at $5 \mathrm{GPa}$ was irradiated by a pulse train separated by $443 \mathrm{~ns}(2.2 \mathrm{MHz})$, the estimated sample temperature at the delay time was above $1400 \mathrm{~K}$, confirmed by in situ transformation of $\alpha$ - to $\gamma$-iron (Fig. 23).

\subsection{Combined spectroscopy and diffraction experiments in DACs}

Differently to IC2, the DAC setup in IC1 was used in a number of measurements that combined emission spectroscopy [at the Fe-emission lines (Lin et al., 2005)] and X-ray diffraction at $13 \mathrm{keV}$ incident XFEL photon energy. The goal of the experiment was to study simultaneously the structure

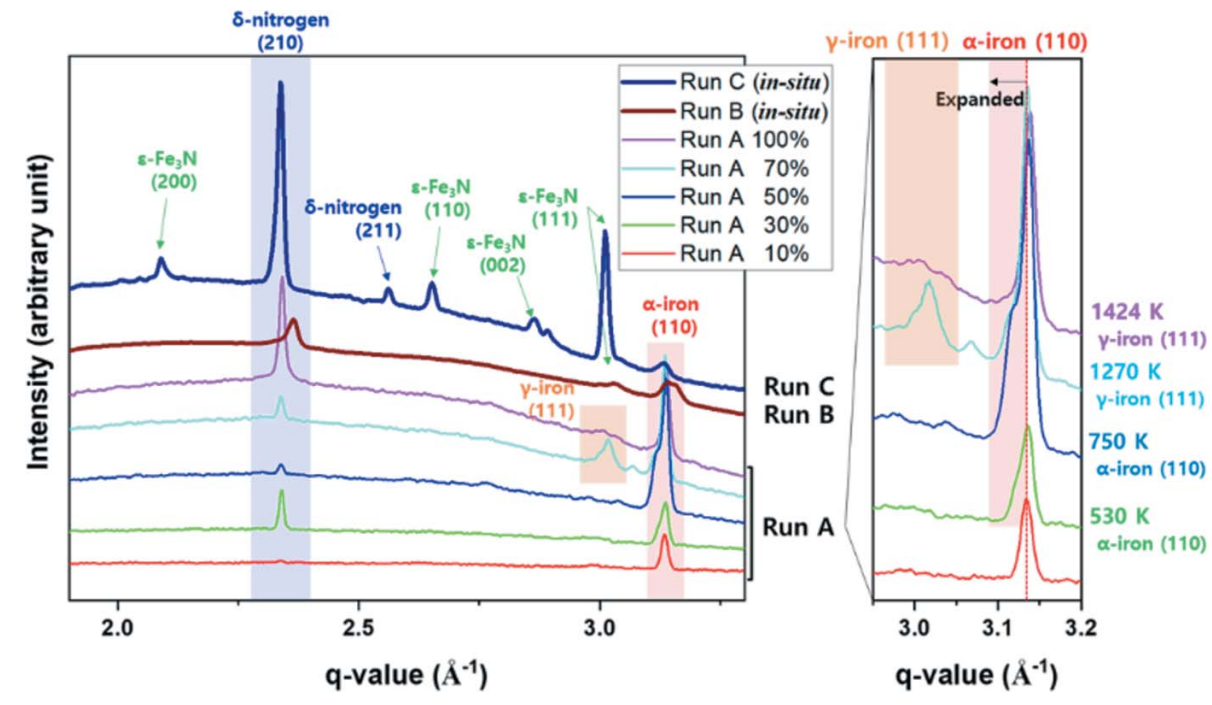

Figure 23

The changes of X-ray diffraction patterns of iron and nitrogen during in situ chemical reaction by XFEL pump-and-probe. An Fe foil surrounded by $\mathrm{N}_{2}$ was pre-compressed to $5 \mathrm{GPa}$ in a DAC. The percentages in run $A$ indicate the transmission (fluence) of the XFEL. At $100 \%$ XFEL transmission, run $B$ contains 20 consecutive pulses while run $C$ has consecutive pulses over $11 \mathrm{~s}$. For more details and figure credit, refer to Hwang et al. (2021). 
and electronic configuration of Fe-bearing compounds at extreme pressures and temperatures in situ. While under pressure, the sample was subjected to trains of several X-ray pulses which step-wise heated the sample in the DAC. During this temperature increase, the sample undergoes a spin crossover (Cerantola et al., 2015), which was detected both in diffraction and emission signals.

\subsection{Laser shock compression experiments on planetary materials}

Geodynamic models of planetary interiors depend on the precise knowledge of the equation of state and the highpressure phase diagram of materials such as $\mathrm{SiO}_{2}$ and $\mathrm{MgO}$. Previous studies demonstrated in shock compression experiments that these materials can transition from electrically insulating solids to conducting liquids at ultra-high pressures (Umemoto et al., 2006; Millot et al., 2015) which suggest, for instance, that planetary structure models need to include multiple layers of conductive fluids.

The use of the DiPOLE 100-X laser system will allow investigating matter at extreme conditions by laser-induced shock- and ramp-compression. It will be possible to investigate the model systems $\mathrm{SiO}_{2}$ and $\mathrm{MgO}$ at pressures of up to several megabars at high or moderate temperatures with temporal pulse-shaping capabilities, which will consequently enable quasi-isentropic compression of material, reaching Hugoniot and off-Hugoniot high-pressure states.

The VISAR and SOP diagnostics will enable measurements of the pressure- and temperature evolution within the sample during shock transit, using a $\mathrm{LiF}$ or $\mathrm{Al}_{2} \mathrm{O}_{3}$ pressure window. Probing the materials during shock loading with the highly coherent and intense X-ray beam will permit the collection of time-resolved information about their respective density and crystal structures during compression.

\subsection{Diagnosing relativistic laser plasmas}

Small-angle X-ray scattering (SAXS) allows the detection of nanoscale density fluctuations. Typically, the SAXS signal from laser-excited plasmas is expected to be dominated by the free-electron distribution (Kluge et al., 2014); however, the fully tunable low energy-bandwidth XFEL pulses can be exploited for resonant SAXS (Kluge et al., 2016). Here, ionic scattering becomes visible when the incoming X-ray photon is in resonance with a bound-bound electronic transition. In this case, the scattering cross-section dramatically increases so that the signal of X-ray scattering from ions is silhouetted against the free-electron scattering background which allows the measurement of opacity and derived quantities with high spatial and temporal resolution.

In the harsh environment of high-intensity laser interactions, intense secondary radiation and high-energy particles are generated, and the SAXS signal may suffer a significant increase of noise. To mitigate the noise, a mosaic graphite crystal that works as a Bragg mirror for the SAXS signal between 8 and $9 \mathrm{keV}$ is developed, which allows detecting the signal behind an appropriate shielding (Šmíd et al., 2020).
In addition, a solid target irradiated by a high-intensity laser pulse can become relativistically transparent, which then allows it to sustain an extremely strong laser-driven longitudinal electron current. The current generates a filament with a slowly varying Mega-Tesla level azimuthal magnetic field that has been shown to prompt efficient emission of multi-MeV photons in the form of a collimated beam required for multiple applications. At the HED instrument, these shortlived transient magnetic fields can be diagnosed via Faraday rotation (Wang et al., 2019).

\subsection{Grazing-incidence X-ray scattering (GIXS) to track surface and subsurface density dynamics at nanometre resolution}

The interaction between high-power, sub-ps lasers with metals or high-density plasmas occurs within the skin depth of the overcritical plasma, where the laser wave is evanescent. Therefore, the laser-plasma coupling relies on the details of the surface nano-structure within the skin layer, which amounts to a few tens of $\mathrm{nm}$ for solids. For a clear understanding of the complex physics involved in laser-matter coupling and subsequent energy transport, phase transition and surface expansion or ablation, it is crucial to visualize the surface and sub-surface density profile with nanometre and sub-ps resolution. X-rays become surface-sensitive in a grazing-incidence geometry, i.e. close to the critical angle for external total reflection which is typically below $1^{\circ}$ for solids in the hard X-ray regime. In particular, grazing-incidence X-ray scattering (GIXS), which blocks the intense specular reflection and looks at the diffusely scattered $2 \mathrm{D}$ pattern, provides a wealth of information about the depth profile as well as the surface roughness and its correlation properties (Holy et al., 1993; Müller-Buschbaum, 2003; Roth, 2016). The first grazingincidence X-ray scattering of a femtosecond XFEL beam combined with a high-intensity femtosecond laser has been successfully demonstrated at the SACLA XFEL in Japan (Randolph et al., 2020).

\subsection{Probing the vacuum polarization}

Vacuum in the presence of strong electromagnetic field contains pairs of short-lived quantum particles that can act as an electric dipole, which may in turn change properties of the background field, a process called vacuum polarization (Karbstein \& Mosman, 2019). To detect vacuum polarization effects, the probing light, in this case the XFEL beam, has to be linearly polarized with a high degree of purity (Schlenvoigt et al., 2016). A specially designed highly optimized channel-cut polarizer and analyzer, each reflecting the beam six times inside the channel, has been shown to suppress residual light polarized in the orthogonal state to more than 11 orders of magnitude (Grabiger et al., 2020; Bernhardt et al., 2020). Such polarization purity is unprecedented for X-ray sources and opens up new opportunities for quantum optics and polarimetric experiments at XFELs. 
Table 7

Overview of X-ray methods supported at the HED scientific instrument.

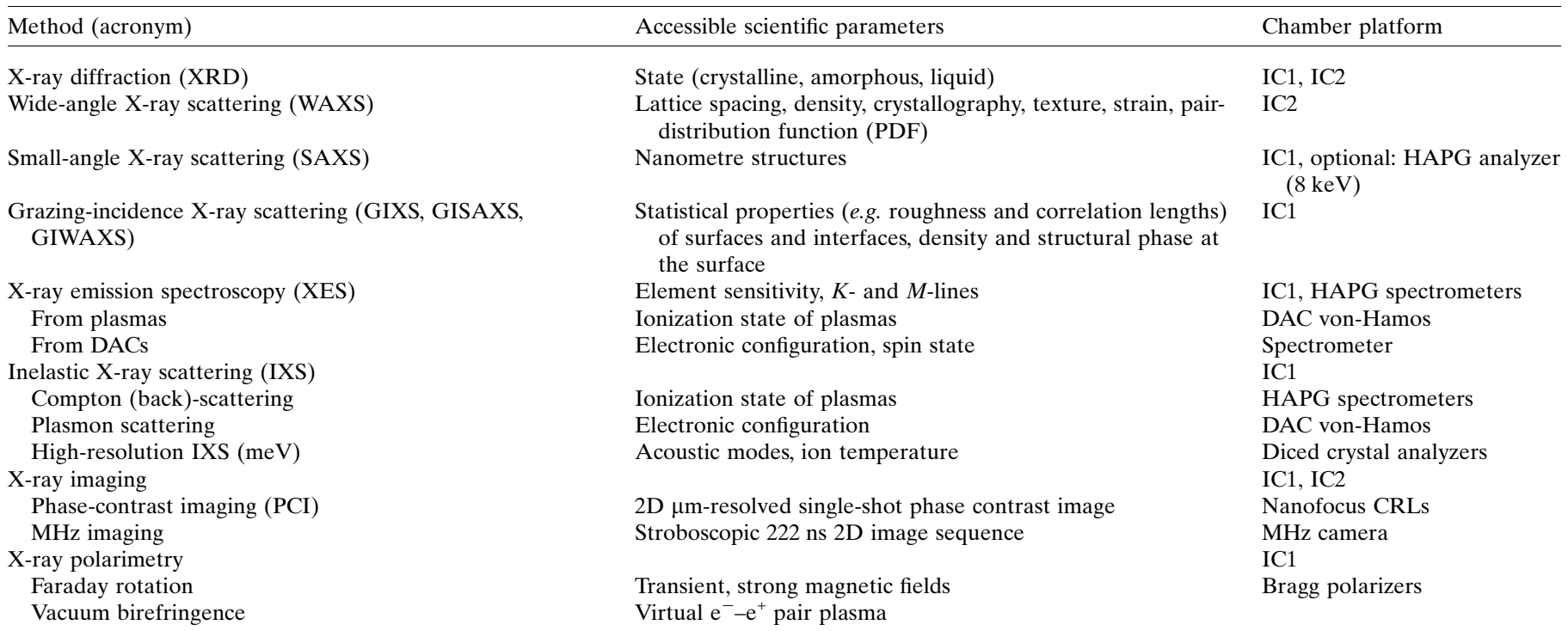

7.7. Structure, electronic, and magnetic properties in pulsed high magnetic fields

One of the open questions in underdoped cuprate high- $T_{\mathrm{c}}$ superconductors is the nature and connection of the different charge density wave states with the upper critical field. To study the emergence of superconductivity from these phases, diffraction from the weak charge density wave peaks in very high magnetic fields is required (Gerber et al., 2015). Indeed, suppressing electronic order may provide a more general route to find new types of superconductors in various families of materials (Basov \& Chubukov, 2011). The suppression of electronic order in high magnetic fields can also result in quantum criticality. Quantum critical matter in high magnetic fields may not only exhibit superconductivity but also electronic nematic phases (e.g. Fe-Pns) or so-called hidden orders, e.g. $\mathrm{URu}_{2} \mathrm{Si}_{2}$ (Mydosh \& Oppeneer, 2011). In frustrated magnetic materials, diffraction allows the detection of broken structural symmetries accommodating a variety of magnetic phases, and magnetic scattering will make it possible to probe their order. Example systems where a magnetic field successively stabilizes a variety of novel exotic states (Ueda et al., 2017) are the rare-earth iridates of the $A_{2} B_{2} \mathrm{O}_{7}$ pyrochlore structure. Here, resonant magnetic X-ray diffraction with polarization control and analysis could be used to probe the interplay between the rare earth and Ir, in order to understand the quantum metal-insulator transition.

\section{Conclusion}

We present the status of the HED instrument at the European XFEL after the first two years of operation. The performance of the baseline instrumentation, short-term future developments, and technical infrastructure are discussed. Experimental platforms and their diagnostics offered at the HED instrument are presented and first scientific results are highlighted.

\section{APPENDIX $A$}

$\mathrm{X}$-ray methods

An overview of the X-ray methods supported at the HED scientific instrument are given in Table 7.

\section{APPENDIX $B$ \\ Technical specifications}

A summary of the most important components with positions is given in Table 8 . The compound refractive lens schemes at HED is shown in Fig. 24, and the configurations of individual lenses are given in Table 9. Properties of X-ray imagers are given in Table 10, and details of the spectral analysers can be found in Table 11.

\section{Acknowledgements}

We acknowledge the European XFEL in Schenefeld, Germany, for provision of X-ray free electron laser beam time at the Scientifc Instrument HED (High Energy Density Science) and would like to thank the staff for their assistance. The authors are indebted to the HIBEF user consortium for the provision of instrumentation and staff that enabled this experiment. The HED Advisory Review Team - consisting of Richard W. Lee (chair), Partick Audebert, Andrew Higginbotham, Hae Ja Lee, Hanns-Peter Liermann, David Neely, Paul Neumayer, Klaus Sokolowski-Tinten and Sven Toleikis are acknowledged for their expert advice and thorough review of the instrument design. We thank S. Pascarelli, Justin S. Wark, Ronald Redmer and Edgar Weckert as members of the HIBEF management board. Science Advisory Committee are also thanked for their advice and feedback on the instrument scope and design. We would like to thank all involved the European XFEL groups - in particular X-ray Optics (XRO), Controls, Detector (DET) and X-Ray Photon Diagnostics (XPD), safety and radiation protection (SRP) - as well as 
Table 8

A summary of the most important components with positions.

The coordinate system at EuXFEL is based on the center of the last undulator U33, which was by design assumed to be the source point.

\begin{tabular}{|c|c|c|c|c|c|c|c|}
\hline XTD1/6 & $\begin{array}{l}\text { Distance } \\
(\mathrm{m})\end{array}$ & XTD6 & $\begin{array}{l}\text { Distance } \\
(\mathrm{m})\end{array}$ & OPT & $\begin{array}{l}\text { Distance } \\
(\mathrm{m})\end{array}$ & EXP & $\begin{array}{l}\text { Distance } \\
(\mathrm{m})\end{array}$ \\
\hline Source point U33 & 0.0 & ABS & 306.6 & OPT hutch wall & 958 & EXP hutch wall & 967.8 \\
\hline Undulator end & 12.2 & $\operatorname{COLA}(65 \mathrm{~mm} \times 25 \mathrm{~mm})$ & 307.6 & PAM center & 960 & ALAS (center) & 968.5 \\
\hline Transmissive imager & 181.1 & HIREX-II & 380.8 & Attenuators center & 961 & TCC IC1 & 971.3 \\
\hline SRA & 188.4 & PBLM & 387.1 & CRL3 center pos. & 962.3 & TCC IC2 & 975.0 \\
\hline KMONO & 190.6 & M3 & 390 & IPM & 965.8 & IBS & 980 \\
\hline COLB (17 mm diameter) & 192.0 & Imager (II-45) & 400 & OPT/EXP wall & 967 & End of EXP hutch & 979.2 \\
\hline XGMin XTD1 & 199.6 & Separation MID/HED & 462.1 & & & & \\
\hline ATT & 227.6 & PBLM & 827.7 & & & & \\
\hline CRL1 & 229 & COLB (18 mm diameter) & 828.5 & & & & \\
\hline FEL Imager & 242 & SDL & 841.0 & & & & \\
\hline XTD1 end/XS2 start & 245 & BIU1 & 844.8 & & & & \\
\hline XS2 end & 277.2 & MONO four-bounce & 853.5 & & & & \\
\hline XTD6 start & 277.2 & HR MONO & 855.8 & & & & \\
\hline M1 & 290 & CRL2 & 857 & & & & \\
\hline PBLM & 298.0 & BIU2 & 858.0 & & & & \\
\hline M2 & 301.4 & $\operatorname{COLB}(14 \mathrm{~mm} \times 24 \mathrm{~mm})$ & 858.6 & & & & \\
\hline $\mathrm{MCP}$ & 302.9 & $\mathrm{XGM}$ in XTD6 & 866.2 & & & & \\
\hline \multirow[t]{5}{*}{ Imager (II-45) } & 303.7 & Pulse picker & 877.7 & & & & \\
\hline & & COLA (30 mm diameter) & 900 & & & & \\
\hline & & Imager (type I) & 939.1 & & & & \\
\hline & & Front end & 940.2 & & & & \\
\hline & & Wall end & 942.6 & & & & \\
\hline
\end{tabular}

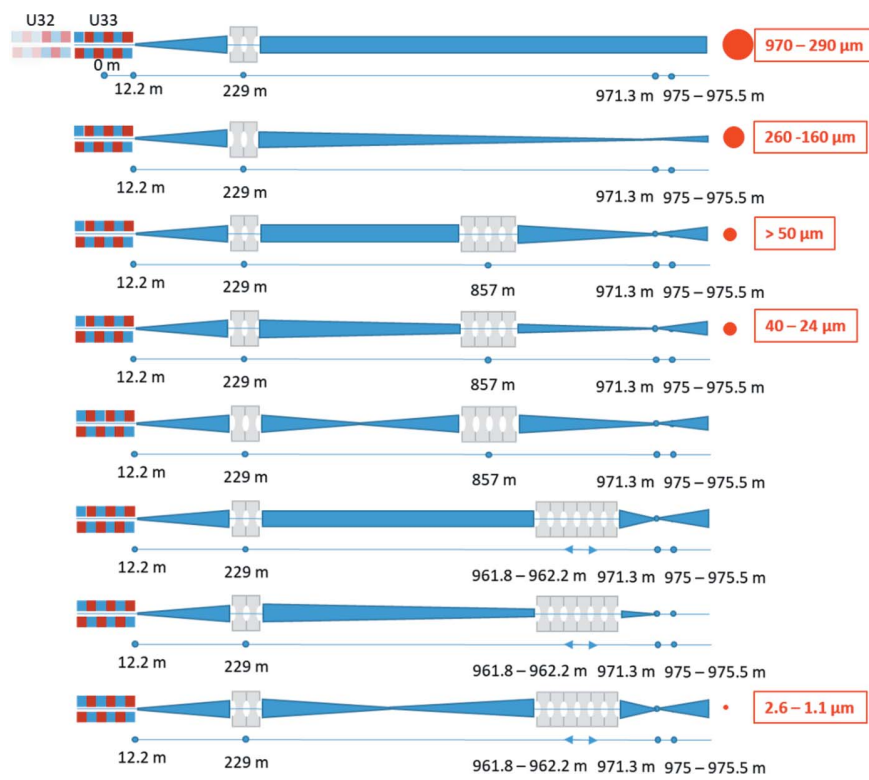

Figure 24

HED focusing optics. The X-rays enter from the left. The coordinate system starts at the center of U33. On the right, in red, are the achievable best focus conditions for $5-25 \mathrm{keV}$ for monochromatic (seeded) beam.

the DESY groups - MKK, MEA, MPS and D3 - for their contributions during the construction phase of the instrument; the members of the photon commissioning team; Naresh Kujala for his work on the HIREX-II spectrometer; Liubov Samoylova for sharing her expertise on the X-ray monochromator; Zunaira Ansari for implementation of radiationsafety measures; Theophilos Maltezopoulos for setting up the XGMs; Istvan Mohacsi for development of the scantool; and Marijan Stupar for PLC-related assistance. Furthermore, we thank Winfried Decking and his team for their support in preparing Fig. 2. We thank Anna Ferrari (HZDR) for FLUKA simulation to determine the concrete thickness of the HED experiment enclosure. HDL at HZDR acknowledges support as member of the European Magnetic Field Laboratory (EMFL) and by DFG excellence cluster ct.qmat (EXC 2147, Project ID 39085490). We gratefully acknowledge the dedication of Gerd Priebe $(\dagger 2017)$ to the instrument, particularly for developing the concept of ReLaX and DiPOLE laser integration as well as the laser infrastructure. We are grateful to Marion Harmand and Philippe Rosier from IMPMC laboratory, CNRS, for their contribution of the CNRS spectrometer (CNRS AAP TGIR 2019). We thank Sakura Pascarelli and the members of the peer-review panel. Open access funding enabled and organized by Projekt DEAL.

\section{Funding information}

Funding for this research was provided by: Deutsche Forschungsgemeinschaft (grant No. FOR 2440; grant No. KO5262/1); Bundesministerium für Bildung und Forschung (grant No. 05K19PE2; grant No. AP 2621-2; grant No. 05K13PM1; grant No. 05K10PM2); Volkswagen Foundation (grant No. Peter-Paul Ewald Fellowship); Centre National de la Recherche Scientifique (grant No. CNRS AAP TGIR 2019).

\section{References}

Abeghyan, S., Bagha-Shanjani, M., Chen, G., Englisch, U., Karabekyan, S., Li, Y., Preisskorn, F., Wolff-Fabris, F., Wuenschel, M., Yakopov, M. \& Pflueger, J. (2019). J. Synchrotron Rad. 26, 302310.

Allahgholi, A., Becker, J., Delfs, A., Dinapoli, R., Göttlicher, P., Graafsma, H., Greiffenberg, D., Hirsemann, H., Jack, S., Klyuev, A., Krüger, H., Kuhn, M., Laurus, T., Marras, A., Mezza, D., 
Table 9

Configurations of individual lenses (number and radii of curvature) in all installed CRL systems.

CRL 1, 2 and 3 consist of a so-called transfocator with ten rods which can be individually inserted. The term number refers to the amount of individual Be lenses in each rod. The apertures of these parabolic lenses relate to the maximum non-clipping illumination. The shown lens configuration in CRL3 can focus all photon energies from $5 \mathrm{keV}$ up to $20.5 \mathrm{keV}$ without any gap. If tight focusing of higher photon energies is required, either prefocusing with CRL1,2 needs to be used, or weaker lenses have to be replaced with stronger ones. However, the latter will result in gaps for focusing lower photon energies.

\begin{tabular}{|c|c|c|c|c|c|c|c|c|c|c|}
\hline Rod & 1 & 2 & 3 & 4 & 5 & 6 & 7 & 8 & 9 & 10 \\
\hline CRL1 lens radius $(\mathrm{mm}) \times$ number & - & $5.8 \times 1$ & $5.0 \times 1$ & $4.0 \times 1$ & $3.5 \times 1$ & $5.8 \times 2$ & $4.0 \times 3$ & $4.0 \times 7$ & $2.0 \times 7$ & - \\
\hline CRL1 apertures $(\mathrm{mm})$ & 4 & 3.8 & 3.53 & 3.16 & 2.96 & 3.8 & 3.16 & 3.16 & 2.76 & 4 \\
\hline CRL2 lens radius $(\mathrm{mm}) \times$ number & $5.8 \times 1$ & $5.0 \times 1$ & $4.0 \times 1$ & $3.5 \times 1$ & $5.8 \times 2$ & $5.8 \times 4$ & $5.8 \times 7$ & $4.0 \times 10$ & $3.5 \times 10$ & $2.0 \times 8$ \\
\hline CRL2 apertures (mm) & 3.8 & 3.53 & 3.16 & 2.96 & 3.8 & 3.8 & 3.8 & 3.16 & 2.96 & 2.76 \\
\hline CRL3 lens radius $(\mathrm{mm}) \times$ number & $5.8 \times 1$ & $5.8 \times 3$ & $5.8 \times 4$ & $4.0 \times 10$ & $2.0 \times 10$ & $1.0 \times 10$ & $1.0 \times 10$ & $0.5 \times 10$ & $0.5 \times 10$ & $5.8 \times 2$ \\
\hline CRL3 apertures $(\mathrm{mm})$ & 3.8 & 3.8 & 3.8 & 3.16 & 2.76 & 1.95 & 1.95 & 1.38 & 1.38 & 3.8 \\
\hline CRL4 lens radius $(\mathrm{mm}) \times$ number & $0.05 \times$ up to 50 & & & & & & & & & \\
\hline CRL4 apertures (mm) & 0.3 & & & & & & & & & \\
\hline
\end{tabular}

Table 10

Summary of each $2 \mathrm{D}$ imager, $10 \mathrm{~Hz}$ operation.

Invasive means that this imager is opaque to the $\mathrm{X}$-ray beam, as opposed to an imager which is transmissive.

\begin{tabular}{|c|c|c|c|c|c|}
\hline 2D imager & $\begin{array}{l}\text { Location from } \\
\text { the source }(\mathrm{m})\end{array}$ & Type & Screens and thicknesses & $\begin{array}{c}\text { Field of view } \\
(\mathrm{mm} \times \mathrm{mm})\end{array}$ & $\begin{array}{l}\text { Resolution } \\
(\mu \mathrm{m})\end{array}$ \\
\hline IMG-TR & 190 & Transmissive & YAG:Ce $25 \mu \mathrm{m}$, diamond $50 \mu \mathrm{m}$ & $25.6 \times 19.2$ & 35 \\
\hline IMGFEL & 242 & Invasive & YAG:Ce $100 \mu \mathrm{m}, \mathrm{BN} 60 \mu \mathrm{m}, \mathrm{CVD} 40 \mu \mathrm{m}$ & $17.62 \times 23.5$ & $15,15,59$ \\
\hline POPInII45-2 & 400 & Transmissive & YAG:Ce $100 \mu \mathrm{m}$ & $148.4 \times 22.1$ & 112 \\
\hline SDLBM-1 & 845 & Transmissive & YAG:Ce $1 \mathrm{~mm}$ & $\gg 63 \mathrm{~mm}$ & $\gg 200$ \\
\hline SDLBM-2 & 858 & Transmissive & YAG:Ce $1 \mathrm{~mm}$ & $\gg 63 \mathrm{~mm}$ & $\gg 200$ \\
\hline HED-SBM & 965 & Transmissive & YAG:Ce $25 \mu \mathrm{m} \& 50 \mu \mathrm{m}$ for beam split by BIU grating & N/A & \\
\hline HED-Questar1 & 971.3 & Transmissive & Variable & $13 \times 8$ & 20 \\
\hline HED-Questar2 & 971.3 & Transmissive & Variable & $13 \times 8$ & 20 \\
\hline HED-IBS & 978.5 & Invasive & CVD $500 \mu \mathrm{m}$ & N/A & \\
\hline
\end{tabular}

Table 11

Summary of each spectral analyser.

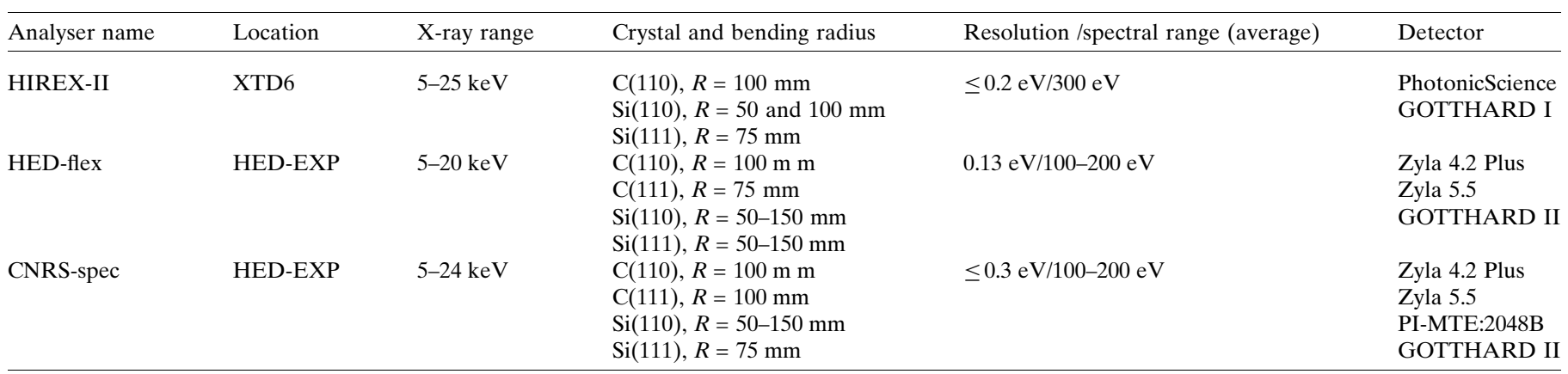

Mozzanica, A., Poehlsen, J., Shefer Shalev, O., Sheviakov, I., Schmitt, B., Schwandt, J., Shi, X., Smoljanin, S., Trunk, U., Zhang, J. \& Zimmer, M. (2019). Nucl. Instrum. Methods Phys. Res. A, 942, 162324.

Appel, K., Nakatsutsumi, M., Pelka, A., Priebe, G., Thorpe, I. \& Tschentscher, T. (2014). Plasma Phys. Control. Fusion, 57, 014003.

Appleby, G. A. S., Pascarelli, D., Pahl, T., Tschentscher (2017). Synchrotron Radiat. News, 30(5), 6-8.

Barker, L. M. \& Hollenbach, R. E. (1965). Rev. Sci. Instrum. 36, 16171620.

Basov, D. N. \& Chubukov, A. V. (2011). Nat. Phys. 7, 272-276.

Battistoni, G., Boehlen, T., Cerutti, F., Chin, P. W., Esposito, L. S., Fassò, A., Ferrari, A., Lechner, A., Empl, A., Mairani, A.,
Mereghetti, A., Ortega, P. G., Ranft, J., Roesler, S., Sala, P. R., Vlachoudis, V. \& Smirnov, G. (2015). Ann. Nucl. Energy, 82, 10-18. Bernhardt, H., Schmitt, A. T., Grabiger, B., Marx-Glowna, B., Loetzsch, R., Wille, H., Bessas, D., Chumakov, A. I., Rüffer, R., Röhlsberger, R., Stöhlker, T., Uschmann, I., Paulus, G. G. \& Schulze, K. S. (2020). Phys. Rev. Res. 2, 023365.

Bionta, M. R., Lemke, H. T., Cryan, J. P., Glownia, J. M., Bostedt, C., Cammarata, M., Castagna, J. C., Ding, Y., Fritz, D. M., Fry, A. R., Krzywinski, J., Messerschmidt, M., Schorb, S., Swiggers, M. L. \& Coffee, R. N. (2011). Opt. Express, 19, 21855-21865.

Blaj, G., Bhogadi, D., Chang, C., Doering, D., Kenney, C., Kroll, T., Segal, J., Sokaras, D. \& Haller, G. (2019). AIP Conf. Proc. 2054, 060037. 
Blaj, G., Caragiulo, P., Dragone, A., Haller, G., Hasi, J., Kenney, C. J., Kwiatkowski, M., Markovic, B., Segal, J. \& Tomada, A. (2016). Proc. SPIE, 9968, 59-68.

Boesenberg, U., Samoylova, L., Roth, T., Zhu, D., Terentyev, S., Vannoni, M., Feng, Y., van Driel, T. B., Song, S., Blank, V., Sinn, H., Robert, A. \& Madsen, A. (2017). Opt. Express, 25, 2852-2862.

Brygoo, S., Millot, M., Loubeyre, P., Lazicki, A. E., Hamel, S., Qi, T., Celliers, P. M., Coppari, F., Eggert, J. H., Fratanduono, D. E., Hicks, D. G., Rygg, J. R., Smith, R. F., Swift, D. C., Collins, G. W. \& Jeanloz, R. (2015). J. Appl. Phys. 118, 195901.

Cerantola, V., McCammon, C., Kupenko, I., Kantor, I., Marini, C., Wilke, M., Ismailova, L., Solopova, N., Chumakov, A., Pascarelli, S. \& Dubrovinsky, L. (2015). Am. Mineral. 100, 2670-2681.

Cerantola, V., Rosa, A. D., Konôpková, Z., Torchio, R., Brambrink, E., Rack, A., Zastrau, U. \& Pascarelli, S. (2021). J. Phys. Condens. Matter, 33, 274003.

Decking, W. et al. (2020). Nat. Photon. 14, 391-397.

Descamps, A., Ofori-Okai, K., Appel, V., Cerantola, A., Comley, J., Eggert, L., Fletcher, D., Gericke, S., Göde, S., Humphries, O., Karnbach, A., Lazicki, R., Loetzsch, D., McGonegle, C., Palmer, C., Plueckthun, T., Preston, R., Redmer, D., Senesky, C., Strohm, I., Uschmann, T., White, L., Wollenweber, G., Monaco, J., Wark, J., Hastings, U., Zastrau, G., Gregori, S., Glenzer, S. H. \& McBride, E. E. (2020). Sci. Rep. 10, 14564.

De Vido, M., Ertel, K., Wojtusiak, A., O'Donoghue, N., Tomlinson, S., Divoky, M., Sawicka-Chyla, M., Pilar, J., Mason, P., Phillips, J., Smith, J. M., Banerjee, S., Butcher, T., Edwards, C., Lucianetti, A., Mocek, T. \& Collier, J. (2019). Advanced Solid State Lasers, p. JTu3A.14. Optical Society of America.

Dong, X., Shu, D. \& Sinn, H. (2016). AIP Conf. Proc. 1741, 040027. Drake, R. P. (2010). Phys. Today, 63, 28-33.

Schneidmiller, E. A. \& Yurkov, M. V. (2011). Photon beam properties at the European XFEL (December (2010). revision). Technical Report. Deutsches Elektronen-Synchrotron (DESY), Hamburg Germany.

Fiuza, F., Swadling, G. F., Grassi, A., Rinderknecht, H. G., Higginson, D. P., Ryutov, D. D., Bruulsema, C., Drake, R. P., Funk, S., Glenzer, S., Gregori, G., Li, C. K., Pollock, B. B., Remington, B. A., Ross, J. S., Rozmus, W., Sakawa, Y., Spitkovsky, A., Wilks, S. \& Park, H. (2020). Nat. Phys. 16, 916-920.

Geindre, J. P., Mysyrowicz, A., Santos, A. D., Audebert, P., Rousse, A., Hamoniaux, G., Antonetti, A., Falliès, F. \& Gauthier, J. C. (1994). Opt. Lett. 19, 1997.

Geloni, G., Anton, J., Blank, V., Decking, W., Dong, X., Karabekyan, S., Kearney, S., Kocharyan, V., La Civita, D., Liu, S., et al. (2019). Proceedings of the 39th International Free-Electron Laser Conference (FEL2019), 26-30 August 2019, Hamburg, Germany, pp. 242245. MOP008.

Geloni, G., Kocharyan, V. \& Saldin, E. (2011). J. Mod. Opt. 58, 13911403.

Gerber, S., Jang, H., Nojiri, H., Matsuzawa, S., Yasumura, H., Bonn, D. A., Liang, R., Hardy, W. N., Islam, Z., Mehta, A., Song, S., Sikorski, M., Stefanescu, D., Feng, Y., Kivelson, S. A., Devereaux, T. P., Shen, Z. -X., Kao, C. -C., Lee, W. -S., Zhu, D. \& Lee, J. S. (2015). Science, 350, 949-952.

Gessler, P., Ali, H., Babies, F., Ballak, K. E., Bamaga, H., Baranasic, B., Bieler, O., Coppola, N., Dornack, K., Eilers, J., et al. (2019). Proceedings of the 17th Biennial International Conference on Accelerator and Large Experimental Physics Control Systems (ICALEPCS2019), 5-11 October 2019, New York, NY, USA, pp. 1154-1560. THAPP05.

Göde, S., Rödel, K., Zeil, R., Mishra, M., Gauthier, M., Brack, T., Kluge, T., MacDonald, M. J., Metzkes, L., Obst, L., Rehwald, M., Ruyer, C., Schlenvoigt, H. P., Schumaker, W., Sommer, P., Cowan, T. E., Schramm, U., Glenzer, S. \& Fiuza, F. (2017). Phys. Rev. Lett. 118, 194801.

Grabiger, B., Marx-Glowna, B., Uschmann, I., Loetzsch, R., Paulus, G. G. \& Schulze, K. S. (2020). Appl. Phys. Lett. 117, 201102.
Grissonnanche, G., Laliberté, S., Dufour-Beauséjour, M., Matusiak, S., Badoux, S., Tafti, F. F., Michon, A., Riopel, O., Cyr-Choinière, O., Baglo, J. C., Ramshaw, B. J., Liang, R., Bonn, D. A., Hardy, W. N., Krämer, S., LeBoeuf, D., Graf, D., Doiron-Leyraud, N. \& Taillefer, L. (2016). Phys. Rev. B, 93, 064513.

Grünert, J., Carbonell, M. P., Dietrich, F., Falk, T., Freund, W., Koch, A., Kujala, N., Laksman, J., Liu, J., Maltezopoulos, T., Tiedtke, K., Jastrow, U. F., Sorokin, A., Syresin, E., Grebentsov, A. \& Brovko, O. (2019). J. Synchrotron Rad. 26, 1422-1431.

Hagemann, J., Vassholz, M., Hoeppe, H., Osterhoff, M., Rosselló, J. M., Mettin, R., Seiboth, F., Schropp, A., Möller, J., Hallmann, J., Kim, C., Scholz, M., Boesenberg, U., Schaffer, R., Zozulya, A., Lu, W., Shayduk, R., Madsen, A., Schroer, C. G. \& Salditt, T. (2021). J. Synchrotron Rad. 28, 52-63.

Hámos, L. von (1934). Annal. Phys. 411, 252-260.

Harmand, M., Coffee, R., Bionta, M. R., Chollet, M., French, D., Zhu, D., Fritz, D. M., Lemke, H. T., Medvedev, N., Ziaja, B., Toleikis, S. \& Cammarata, M. (2013). Nat. Photon. 7, 215-218.

Hauf, S., Heisen, B., Aplin, S., Beg, M., Bergemann, M., Bondar, V., Boukhelef, D., Danilevsky, C., Ehsan, W., Essenov, S., Fabbri, R., Flucke, G., Fulla Marsa, D., Göries, D., Giovanetti, G., Hickin, D., Jarosiewicz, T., Kamil, E., Khakhulin, D., Klimovskaia, A., Kluyver, T., Kirienko, Y., Kuhn, M., Maia, L., Mamchyk, D., Mariani, V., Mekinda, L., Michelat, T., Münnich, A., Padee, A., Parenti, A., Santos, H., Silenzi, A., Teichmann, M., Weger, K., Wiggins, J., Wrona, K., Xu, C., Youngman, C., Zhu, J., Fangohr, H. \& Brockhauser, S. (2019). J. Synchrotron Rad. 26, 1448-1461.

Heisen, B., Boukhelef, D., Esenov, S., Hauf, S., Kozlova, I., Maia, L., Parenti, A., Szuba, J., Weger, K., Wrona, K. \& Youngman, C. (2013). Proceedings of the 14th International Conference on Accelerator and Large Experimental Physics Control Systems (ICALEPCS2013), 6-11 October 2013, San Francisco, CA, USA, pp. 1465-1468. FRCOAAB02.

Holy, V., Kuběna, I., Ohlidal, I., Lischka, W. \& Plotz, W. (1993). Phys. Rev. B, 47, 15896-15903.

Hwang, H., Kim, T., Cynn, H., Vogt, T., Husband, R. J., Appel, K., Baehtz, C., Ball, O. B., Baron, M. A., Briggs, R., Bykov, M., Bykova, E., Cerantola, V., Chantel, J., Coleman, A. L., Dattlebaum, D., Dresselhaus-Marais, L. E., Eggert, J. H., Ehm, L., Evans, W. J., Fiquet, G., Frost, M., Glazyrin, K., Goncharov, A. F., Jenei, Z., Kim, J., Konôpková, Z., Mainberger, J., Makita, M., Marquardt, H., McBride, E. E., McHardy, J. D., Merkel, S., Morard, G., O'Bannon, E. F. III, Otzen, C., Pace, E. J., Pelka, A., Pépin, C. M., Pigott, J. S., Prakapenka, V. B., Prescher, C., Redmer, R., Speziale, S., Spiekermann, G., Strohm, C., Sturtevant, B. T., Velisavljevic, N., Wilke, M., Yoo, C., Zastrau, U., Liermann, H., McMahon, M. I., McWilliams, R. S. \& Lee, Y. (2021). J. Phys. Chem. Lett. 12, 32463252.

Jenei, Z., Liermann, H. P., Husband, R., Méndez, A. S. J., Pennicard, D., Marquardt, H., O'Bannon, E. F., Pakhomova, A., Konopkova, Z., Glazyrin, K., Wendt, M., Wenz, S., McBride, E. E., Morgenroth, W., Winkler, B., Rothkirch, A., Hanfland, M. \& Evans, W. J. (2019). Rev. Sci. Instrum. 90, 065114.

Karbstein, F. \& Mosman, E. A. (2019). Phys. Rev. D, 100, 033002.

Kärcher, V., Roling, S., Samoylova, L., Buzmakov, A., Zastrau, U., Appel, K., Yurkov, M., Schneidmiller, E., Siewert, F. \& Zacharias, H. (2021). J. Synchrotron Rad. 28, 350-361.

Kim, J. B., Göde, S. \& Glenzer, S. H. (2016). Rev. Sci. Instrum. 87, $11 \mathrm{E} 328$.

Kirkwood, H. J., Letrun, R., Tanikawa, T., Liu, J., Nakatsutsumi, M., Emons, M., Jezynski, T., Palmer, G., Lederer, M., Bean, R., Buck, J., Di Dio Cafisio, S., Graceffa, R., Grünert, J., Göde, S., Höppner, H., Kim, Y., Konopkova, Z., Mills, G., Makita, M., Pelka, A., Preston, T. R., Sikorski, M., Takem, C. M. S., Giewekemeyer, K., Chollet, M., Vagovic, P., Chapman, H. N., Mancuso, A. P. \& Sato, T. (2019). Opt. Lett. 44, 1650-1653. 
Klačková, I., Blaj, G., Denes, P., Dragone, A., Göde, S., Hauf, S., Januschek, F., Joseph, J. \& Kuster, M. (2019). J. Instrum. 14, C01008.

Klementiev, K. \& Chernikov, R. (2020). kklmn/xrt: Release 1.3.4, https://doi.org/10.5281/zenodo.3838709.

Kluge, T., Bussmann, M., Chung, H., Gutt, C., Huang, L. G., Zacharias, M., Schramm, U. \& Cowan, T. E. (2016). Phys. Plasmas, 23, 033103.

Kluge, T., Gutt, C., Huang, L. G., Metzkes, J., Schramm, U., Bussmann, M. \& Cowan, T. E. (2014). Phys. Plasmas, 21, 033110.

Koch, A., Risch, J., Freund, W., Maltezopoulos, T., Planas, M. \& Grünert, J. (2019). J. Synchrotron Rad. 26, 1489-1495.

Konôpková, Z., McWilliams, R. S., Gómez-Pérez, N. \& Goncharov, A. F. (2016). Nature, 534, 99-101.

Kujala, N., Freund, W., Liu, J., Koch, A., Falk, T., Planas, M., Dietrich, F., Laksman, J., Maltezopoulos, T., Risch, J., Dall'Antonia, F. \& Grünert, J. (2020). Rev. Sci. Instrum. 91, 103101.

Lengeler, B., Schroer, C., Tümmler, J., Benner, B., Richwin, M., Snigirev, A., Snigireva, I. \& Drakopoulos, M. (1999). J. Synchrotron Rad. 6, 1153-1167.

Liermann, H. P., Konôpková, Z., Appel, K., Prescher, C., Schropp, A., Cerantola, V., Husband, R. J., McHardy, J. D., McMahon, M. I., McWilliams, R. S., Pépin, C. M., Mainberger, J., Roeper, M., Berghäuser, A., Damker, H., Talkovski, P., Foese, M., Kujala, N., Ball, O. B., Baron, M. A., Briggs, R., Bykov, M., Bykova, E., Chantel, J., Coleman, A. L., Cynn, H., Dattelbaum, D., Dresselhaus-Marais, L. E., Eggert, J. H., Ehm, L., Evans, W. J., Fiquet, G., Frost, M., Glazyrin, K., Goncharov, A. F., Hwang, H., Jenei, Z., Kim, J.-Y., Langenhorst, F., Lee, Y., Makita, M., Marquardt, H., McBride, E. E., Merkel, S., Morard, G., O'Bannon, E. F., Otzen, C., Pace, E. J., Pelka, A., Pigott, J. S., Prakapenka, V. B., Redmer, R., Sanchez-Valle, C., Schoelmerich, M., Speziale, S., Spiekermann, G., Sturtevant, B. T., Toleikis, S., Velisavljevic, N., Wilke, M., Yoo, C.-S., Baehtz, C., Zastrau, U. \& Strohm, C. (2021). J. Synchrotron Rad. 28, 688-706.

Lin, J. F., Struzhkin, V. V., Jacobsen, S. D., Hu, M. Y., Chow, P., Kung, J., Liu, H., Mao, H. K. \& Hemley, R. J. (2005). Nature, 436, 377-380.

Liu, J. Y., Hu, J., Zhang, Q., Graf, D., Cao, H. B., Radmanesh, S. M. A., Adams, D. J., Zhu, Y. L., Cheng, G. F., Liu, X., Phelan, W. A., Wei, J., Jaime, M., Balakirev, F., Tennant, D. A., DiTusa, J. F., Chiorescu, I., Spinu, L. \& Mao, Z. Q. (2017). Nat. Mater. 16, 905-910.

Liu, S., Decking, W., Kocharyan, V., Saldin, E., Serkez, S., Shayduk, R., Sinn, H. \& Geloni, G. (2019). Phys. Rev. Accel. Beams, 22, 060704.

Loubeyre, P., Brygoo, J., Eggert, J., Celliers, P. M., Spaulding, D. K., Rygg, J. R., Boehly, T. R., Collins, G. W. \& Jeanloz, R. (2012). Phys. Rev. B, 86, 144115 .

Lu, W., Friedrich, B., Noll, T., Zhou, J., Hallmann, G., Ansaldi, T., Roth, T., Serkez, S., Geloni, G., Madsen, A. \& Eisebitt, S. (2018). Rev. Sci. Instrum. 89, 063121.

Madsen, A., Hallmann, J., Ansaldi, G., Roth, T., Lu, W., Kim, C., Boesenberg, U., Zozulya, A., Möller, J., Shayduk, R., Scholz, M., Bartmann, A., Schmidt, A., Lobato, I., Sukharnikov, K., Reiser, M., Kazarian, K. \& Petrov, I. (2021). J Synchrotron Rad, 28, 637-649.

Maltezopoulos, T., Dietrich, F., Freund, W., Jastrow, U. F., Koch, A., Laksman, J., Liu, J., Planas, M., Sorokin, A. A., Tiedtke, K. \& Grünert, J. (2019). J. Synchrotron Rad. 26, 1045-1051.

Meza-Galvez, J., Gomez-Perez, N., Marshall, A. S., Coleman, A. L., Appel, K., Liermann, H. P., McMahon, M. I., Konôpková, Z. \& McWilliams, R. S. (2020). J. Appl. Phys. 127, 195902.

Millot, M., Dubrovinskaia, N., ernok, A., Blaha, S., Dubrovinsky, L., Braun, D. G., Celliers, P. M., Collins, G. W., Eggert, J. H. \& Jeanloz, R. (2015). Science, 347, 418-420.

Mitzner, R., Siemer, B., Neeb, M., Noll, T., Siewert, F., Roling, S., Rutkowski, M., Sorokin, A. A., Richter, M., Juranic, P., Tiedtke, K., Feldhaus, J., Eberhardt, W. \& Zacharias, H. (2008). Opt. Express, 16, 19909-19919.
Mozzanica, A., Andrä, M., Barten, R., Bergamaschi, A., Chiriotti, S., Brückner, M., Dinapoli, R., Fröjdh, E., Greiffenberg, D., Leonarski, F., Lopez-Cuenca, C., Mezza, D., Redford, S., Ruder, C., Schmitt, B., Shi, X., Thattil, D., Tinti, G., Vetter, S. \& Zhang, J. (2018). Synchrotron Radiat. News, 31(6), 16-20.

Mozzanica, A., Bergamaschi, A., Dinapoli, R., Graafsma, H., Greiffenberg, D., Henrich, B., Johnson, I., Lohmann, M., Valeria, R., Schmitt, B. \& Xintian, S. (2012). J. Instrum. 7, C01019.

Müller-Buschbaum, P. (2003). Anal. Bioanal. Chem. 376, 3-10.

Mydosh, J. A. \& Oppeneer, P. M. (2011). Rev. Mod. Phys. 83, 13011322.

Nakatsutsumi, M., Appel, C., Baehtz, B., Chen, B., Cowan, S., Göde, Z., Konopkova, A., Pelka, G., Priebe, A., Schmidt, K., Sukharnikov, I., Thorpe, Th., Tschentscher, T. \& Zastrau, U. (2016). Plasma Phys. Control. Fusion, 59, 014028.

Nakatsutsumi, M., Appel, K., Priebe, G., Thorpe, I., Pelka, A., Muller, B. \& Tschentscher, Th. (2014). Scientific Instrument High Energy Density Physics (HED). Conceptual Design Report: European Xray Free-Electron Laser Facility GmbH, Schenefeld, Germany.

Nakatsutsumi, M. \& Tschentscher, Th. (2013). Scientific Instrument High Energy Density Physics (HED). Technical Design Report. European X-ray Free-Electron Laser Facility GmbH, Schenefeld, Germany.

Obst, L., Göde, S., Rehwald, M., Brack, F., Branco, J., Bock, S., Bussmann, M., Cowan, T. E., Curry, C. B., Fiuza, F., Gauthier, M., Gebhardt, R., Helbig, U., Huebl, A., Hübner, U., Irman, A., Kazak, L., Kim, J. B., Kluge, T., Kraft, S., Loeser, M., Metzkes, J., Mishra, R., Rödel, C., Schlenvoigt, H., Siebold, M., Tiggesbäumker, J., Wolter, S., Ziegler, T., Schramm, U., Glenzer, S. H. \& Zeil, K. (2017). Sci. Rep. 7, 10248.

Palmer, G., Kellert, M., Wang, J., Emons, M., Wegner, U., Kane, D., Pallas, F., Jezynski, T., Venkatesan, S., Rompotis, D., Brambrink, E., Monoszlai, B., Jiang, M., Meier, J., Kruse, K., Pergament, M. \& Lederer, M. J. (2019). J. Synchrotron Rad. 26, 328-332.

Phillips, J. P., Banerjee, S., Mason, P., Smith, J., Spear, J., De Vido, M., Ertel, K., Butcher, T., Quinn, G., Clarke, D., Edwards, C., Hernandez-Gomez, C. \& Collier, J. (2021). Opt. Lett. 46, 1808.

Phillips, P. J., Mason, P., Ertel, K., Smith, J., De-Vido, M., Butcher, T., Tomlinson, S., Suarez-Merchan, J. E., Lintern, A., Costello, B., Hollingham, I., Norton, A., Tyldesley, M., Hernandez-Gomez, C., Edwards, C., Collier, J., Höppner, H., Toncian, T., Zastrau, U. \& Möller, D. (2019). Proc. SPIE, 10898, 108980K.

Pikuz, T., Faenov, A., Matsuoka, T., Matsuyama, S., Yamauchi, K., Ozaki, N., Albertazzi, B., Inubushi, Y., Yabashi, M., Tono, K., Sato, Y., Yumoto, H., Ohashi, H., Pikuz, S., Grum-Grzhimailo, A. N., Nishikino, M., Kawachi, T., Ishikawa, T. \& Kodama, R. (2015). Sci. Rep. 5, 17713.

Prencipe, I., Fuchs, J., Pascarelli, S., Schumacher, D. W., Stephens, R. B., Alexander, N. B., Briggs, R., Büscher, M., Cernaianu, M. O., Choukourov, A., De Marco, M., Erbe, A., Fassbender, J., Fiquet, G., Fitzsimmons, P., Gheorghiu, C., Hund, J., Huang, L. G., Harmand, M., Hartley, N. J., Irman, A., Kluge, T., Konopkova, Z., Kraft, S., Kraus, D., Leca, V., Margarone, D., Metzkes, J., Nagai, K., Nazarov, W., Lutoslawski, P., Papp, D., Passoni, M., Pelka, A., Perin, J. P., Schulz, J., Smid, M., Spindloe, C., Steinke, S., Torchio, R., Vass, C., Wiste, T., Zaffino, R., Zeil, K., Tschentscher, T., Schramm, U. \& Cowan, T. E. (2017). High Pow Laser Sci Eng, 5, e17.

Preston, T. R., Göde, S., Schwinkendorf, J., Appel, K., Brambrink, E., Cerantola, V., Höppner, H., Makita, M., Pelka, A., Prescher, C., Sukharnikov, K., Schmidt, A., Thorpe, I., Toncian, T., Amouretti, A., Chekrygina, D., Falcone, R. W., Falk, K., Fletcher, L. B., Galtier, E., Harmand, M., Hartley, N. J., Hau-Riege, S. P., Heimann, P., Huang, L. G., Humphries, O. S., Karnbach, O., Kraus, D., Lee, H. J., Nagler, B., Ren, S., Schuster, A. K., Smid, M., Voigt, K., Zhang, M. \& Zastrau, U. (2020). J. Instrum. 15, P11033.

Randolph, L., Banjafar, M., Preston, T. R., Yabuuchi, T., Makita, M., Dover, N. P., Rödel, C., Göde, S., Inubushi, Y., Jakob, G., Kaa, J.,. Kon, A., Koga, J. K., Ksenzov, D., Matsuoka, T., Nishiuchi, M., 
Paulus, M., Schon, F., Sueda, K., Sentoku, Y., Togashi, T., VafaeeKhanjani, M., Bussmann, M., Cowan, T. E., Kläui, M., FortmannGrote, C., Mancuso, A. P., Kluge, T., Gutt, C. \& Nakatsutsumi, M. (2020). arXiv: 2012.15076 [physics. plasm-ph].

Redford, S., Andrä, M., Barten, R., Bergamaschi, A., Brückner, M., Chiriotti, S., Dinapoli, R., Fröjdh, E., Greiffenberg, D., Kim, K. S., Lee, J. H., Lopez-Cuenca, C., Meyer, M., Mezza, D., Mozzanica, A., Park, S., Ruder, C., Schmitt, B., Shi, X., Thattil, D., Tinti, G., Vetter, S. \& Zhang, J. (2020). J. Instrum. 15, C02025.

Redford, S., Andrä, M., Barten, R., Bergamaschi, A., Brückner, M., Dinapoli, R., Fröjdh, E., Greiffenberg, D., Lopez-Cuenca, C., Mezza, D., Mozzanica, A., Ramilli, M., Ruat, M., Ruder, C., Schmitt, B., Shi, X., Thattil, D., Tinti, G., Vetter, S. \& Zhang, J. (2018). J. Instrum. 13, C01027.

Riedel, R., Al-Shemmary, A., Gensch, M., Golz, T., Harmand, M., Medvedev, N., Prandolini, M. J., Sokolowski-Tinten, K., Toleikis, S., Wegner, U., Ziaja, B., Stojanovic, N. \& Tavella, F. (2013). Nat. Commun. 4, 1731.

Roling, S., Appel, K., Braun, S., Buzmakov, A., Chubar, O., Gawlitza, P., Samoylova, L., Siemer, B., Schneidmiller, E., Sinn, H., Siewert, F., Tschentscher, T., Wahlert, F., Wöstmann, M., Yurkov, M. \& Zacharias, H. (2017). Proc. SPIE, 9210, 92100B.

Roth, S. V. (2016). J. Phys. Condens. Matter, 28, 403003.

Ruyer, C., Bolaños, S., Albertazzi, B., Chen, S. N., Antici, P., Böker, J., Dervieux, V., Lancia, L., Nakatsutsumi, M., Romagnani, L., Shepherd, R., Swantusch, M., Borghesi, M., Willi, O., Pépin, H., Starodubtsev, M., Grech, M., Riconda, C., Gremillet, L. \& Fuchs, J. (2020). Nat. Phys. 16, 983-988.

Samoylova, L., Boesenberg, U., Chumakov, A., Kaganer, V., Petrov, I., Roth, T., Rüffer, R., Sinn, H., Terentyev, S. \& Madsen, A. (2019). J. Synchrotron Rad. 26, 1069-1072.

Schlenvoigt, H., Heinzl, T., Schramm, U., Cowan, T. E. \& Sauerbrey, R. (2016). Phys. Scr. 91, 023010.

Schmidt, A. \& Dommach, M. (2015). HV Guidelines for Scientific Instruments. Technical Report. European X-ray Free-Electron Laser Facility GmbH, Schenefeld, Germany.

Scholz, M. \& Zhao, Z. T. (2019). Proceedings of the 39th International Free-Electron Laser Conference (FEL2019), 26-30 August 2019, Hamburg, Germany. MOA04.

Schropp, A., Hoppe, R., Meier, V., Patommel, J., Seiboth, F., Lee, H. J., Nagler, B., Galtier, E. C., Arnold, B., Zastrau, U., Hastings, J. B., Nilsson, D., Uhlén, F., Vogt, U., Hertz, H. M. \& Schroer, C. G. (2013). Sci. Rep. 3, 1633.

Schropp, A., Hoppe, R., Meier, V., Patommel, J., Seiboth, F., Ping, Y., Hicks, D. G., Beckwith, M. A., Collins, G. W., Higginbotham, A., Wark, J. S., Lee, H. J., Nagler, B., Galtier, E. C., Arnold, B., Zastrau, U., Hastings, J. B. \& Schroer, C. G. (2015). Sci. Rep. 5, 11089.

Schropp, A., Patommel, J., Seiboth, F., Arnold, B., Galtier, E. C., Lee, H. J., Nagler, B., Hastings, J. B. \& Schroer, C. G. (2012). Proc. SPIE, 8504, 73-79.

Sinn, H., Dommach, M., Dickert, B., Di Felice, M., Dong, X., Eidam, J., Finze, D., Freijo-Martin, I., Gerasimova, N., Kohlstrunk, N., La Civita, D., Meyn, F., Music, V., Neumann, M., Petrich, M., Rio, B., Samoylova, L., Schmidtchen, S., Störmer, M., Trapp, A., Vannoni, M., Villanueva, R. \& Yang, F. (2019). J. Synchrotron Rad. 26, 692699.

Šmíd, M., Baehtz, A., Pelka, A., Laso García, S., Göde, J., Grenzer, T., Kluge, Z., Konopkova, M., Makita, I., Prencipe, I., Preston, T. R., Rödel, M. \& Cowan, T. E. (2020). Rev. Sci. Instrum. 91, 123501.

Sorokin, A. A., Bican, Y., Bonfigt, S., Brachmanski, M., Braune, M., Jastrow, U. F., Gottwald, A., Kaser, H., Richter, M. \& Tiedtke, K. (2019). J. Synchrotron Rad. 26, 1092-1100.

Sperling, P., Gamboa, E. J., Lee, H. J., Chung, H. K., Galtier, Y., Omarbakiyeva, Y., Reinholz, H., Röpke, U., Zastrau, J., Hastings, J., Fletcher, L. B. \& Glenzer, S. H. (2015). Phys. Rev. Lett. 115, 115001. Spiekermann, G., Kupenko, I., Petitgirard, S., Harder, M., Nyrow, A., Weis, C., Albers, C., Biedermann, N., Libon, L., Sahle, C. J., Cerantola, V., Glazyrin, K., Konôpková, Z., Sinmyo, R., Morgen- roth, W., Sergueev, I., Yavaş, H., Dubrovinsky, L., Tolan, M., Sternemann, C. \& Wilke, M. (2020). J. Synchrotron Rad. 27, 414424.

Tschentscher, T., Bressler, C., Grünert, J., Madsen, A., Mancuso, A., Meyer, M., Scherz, A., Sinn, H. \& Zastrau, U. (2017). Appl. Sci. 7, 592.

Ueda, K., Oh, T., Yang, B. J., Kaneko, R., Fujioka, J., Nagaosa, N. \& Tokura, Y. (2017). Nat. Commun. 8, 15515.

Umemoto, K., Wentzcovitch, R. M. \& Allen, P. B. (2006). Science, 311, 983-986.

Vannoni, M., Freijo Martín, I., Schmidtchen, S., Baumann, T. M., Meyer, M. \& Music, V. (2019). J. Synchrotron Rad. 26, 1110-1114.

Vinko, S. M., Ciricosta, O., Cho, B. I., Engelhorn, K., Chung, H., Brown, C. R. D., Burian, J., Chalupský, J., Falcone, R. W., Graves, C., Hájková, V., Higginbotham, A., Juha, L., Krzywinski, J., Lee, H. J., Messerschmidt, M., Murphy, C. D., Ping, Y., Scherz, A., Schlotter, W., Toleikis, S., Turner, J. J., Vysin, L., Wang, T., Wu, B., Zastrau, U., Zhu, D., Lee, R. W., Heimann, P. A., Nagler, B. \& Wark, J. S. (2012). Nature, 482, 59-62.

Wang, T., Toncian, T., Wei, M. S. \& Arefiev, A. V. (2019). Phys. Plasmas, 26, 013105.

Weise, H. \& Decking, W. (2017). Proceedings of the International Free Electron Laser Conference (FEL'17), 20-25 August 2017, Santa Fe, NM, USA, pp. 9-13. MOC03.

Wheeler, J. A., Borot, A., Monchocé, S., Vincenti, H., Ricci, A., Malvache, A., Lopez-Martens, R. \& Quéré, F. (2012). Nat. Photon. 6, 829-833.

Wilks, S. C., Langdon, A. B., Cowan, T. E., Roth, M., Singh, M., Hatchett, S., Key, M. H., Pennington, D., MacKinnon, A. \& Snavely, R. A. (2001). Phys. Plasmas, 8, 542-549.

Wollenweber, L., Preston, T. R., Descamps, A., Cerantola, V., Comley, A., Eggert, J. H., Fletcher, L. B., Geloni, G., Gericke, D. O., Glenzer, S. H., Göde, S., Hastings, J., Humphries, O. S., Jenei, A., Karnbach, O., Konopkova, Z., Loetzsch, R., Marx-Glowna, B., McBride, E. E., McGonegle, D., Monaco, G., Ofori-Okai, B. K., Palmer, C. A. J., Plückthun, C., Redmer, R., Strohm, C., Thorpe, I., Tschentscher, T., Uschmann, I., Wark, J. S., White, T. G., Appel, K., Gregori, G. \& Zastrau, U. (2021). Rev. Sci. Instrum. 92, 013101.

Wosnitza, J., Zvyagin, S. A. \& Zherlitsyn, S. (2016). Rep. Prog. Phys. 79, 074504 .

Wöstmann, M., Mitzner, T., Noll, S., Roling, B., Siemer, F., Siewert, S., Eppenhoff, F., Wahlert, F. \& Zacharias, H. (2013). J. Phys. B At. Mol. Opt. Phys. 46, 164005.

Yoneda, H., Inubushi, Y., Nagamine, K., Michine, Y., Ohashi, H., Yumoto, H., Yamauchi, K., Mimura, H., Kitamura, H., Katayama, T., Ishikawa, T. \& Yabashi, M. (2015). Nature, 524, 446-449.

Zastrau, U., Audebert, P., Bernshtam, V., Brambrink, E., Kämpfer, T., Kroupp, E., Loetzsch, R., Maron, Y., Ralchenko, Y., Reinholz, H., Röpke, G., Sengebusch, A., Stambulchik, E., Uschmann, I., Weingarten, L. \& Förster, E. (2010). Phys. Rev. E, 81, 026406.

Zastrau, U., Brown, C. R. D., Döppner, T., Glenzer, S. H., Gregori, G., Lee, H. J., Marschner, H., Toleikis, S., Wehrhan, O. \& Förster, E. (2012). J. Instrum. 7, P09015.

Zastrau, U., Woldegeorgis, A., Förster, E., Loetzsch, R., Marschner, H. \& Uschmann, I. (2013). J. Instrum. 8, P10006.

Zastrau, U., McMahon, M., Appel, K., Baehtz, C., Brambrink, E., Briggs, R., Butcher, T., Cauble, B., Chen, B. \& Damker, H. (2017). Dynamic Laser Compression Experiments at the HED Instrument of European XFEL. Conceptual Design Report. European X-ray Free-Electron Laser Facility GmbH, Schenefeld, Germany.

Zhu, D., Cammarata, M., Feldkamp, J. M., Fritz, D. M., Hastings, J. B., Lee, S., Lemke, H. T., Robert, A., Turner, J. L. \& Feng, Y. (2012). Appl. Phys. Lett. 101, 034103.

Zozulya, A., Batchelor, L., Appel, K., Boesenberg, U., Hallmann, J., Kim, C., Lobato, I., Lu, W., Mammen, C., Möller, J., Roth, T., Samoylova, L., Scholz, M., Shayduk, R., Sukharnikov, K. \& Madsen, A. (2019). Proc. SPIE, 11111, 111110H. 